TRANSACTIONS OF THE

AMERICAN MATHEMATICAL SOCIETY

Volume 365, Number 11, November 2013, Pages 5933-5966

S 0002-9947(2013)05825-4

Article electronically published on July 10, 2013

\title{
NORMALLY HYPERBOLIC INVARIANT MANIFOLDS FOR RANDOM DYNAMICAL SYSTEMS: PART I - PERSISTENCE
}

\author{
JI LI, KENING LU, AND PETER BATES
}

ABSTRACT. In this paper, we prove the persistence of smooth normally hyperbolic invariant manifolds for dynamical systems under random perturbations.

\section{INTRODUCTION}

Invariant manifolds provide large-scale structures that serve to organize the global phase space of a dynamical system. Persistence of invariant manifolds under small perturbation, when that occurs, gives a more global robustness or structural stability, allowing one to classify the behavior of families of systems, even those with the vector field known only approximately. Persistence results are particularly important in geometric singular perturbation theory, which provides a powerful technique for analyzing systems involving multiple time scales which arise in applications. The theory gives a rigorous and well-defined way to reduce systems with small parameters to lower-dimensional systems that are more easily analyzed. That theory is well developed for deterministic systems [F1, F2, F3, JK, B, S2] and has been enormously effective in a wide variety of applications (see for instance [J], [BFGJ], [S1], and [KSS]).

This work is the first step in a program to build geometric singular perturbation theory under stochastic perturbations by providing the persistence theory for random normally hyperbolic invariant manifolds. We also give persistence results for random overflowing and random inflowing invariant manifolds, thus extending Fenichel's fundamental results [F1] to randomly perturbed dynamical systems.

Let $(\Omega, \mathcal{F}, P)$ be a probability space and $\left(\theta^{t}\right)_{t \in \mathbb{R}}$ be a measurable $P$-measure preserving dynamical system on $\Omega$. A discrete time random dynamical system (or a cocycle) on space $\mathbb{R}^{n}$ over the dynamical system $\theta^{t}$ is a measurable map

$$
\phi(\cdot, \cdot, \cdot): \mathbb{R} \times \Omega \times \mathbb{R}^{n} \rightarrow \mathbb{R}^{n}, \quad(\omega, x) \mapsto \phi(t, \omega, x), \quad \text { for } t \in \mathbb{R}
$$

such that the map $\phi(t, \omega):=\phi(t, \omega, \cdot)$ forms a cocycle over $\theta^{t}$ :

$$
\begin{gathered}
\phi(0, \omega)=I d, \quad \text { for all } \omega \in \Omega, \\
\phi(t+s, \omega)=\phi\left(t, \theta^{s} \omega\right) \phi(s, \omega), \quad \text { for all } t, s \in \mathbb{R}, \quad \omega \in \Omega .
\end{gathered}
$$

Received by the editors August 30, 2011 and, in revised form, February 28, 2012.

2010 Mathematics Subject Classification. Primary 34C37, 34C45, 34F05, 37H10.

Key words and phrases. Random dynamical systems, random normally hyperbolic invariant manifolds, overflowing manifolds, inflowing manifolds, and random stable and unstable foliations.

The second author was partially supported by NSF0908348.

The third author was partially supported by NSF0909400. 
When $\phi(\cdot, \omega, \cdot): \mathbb{R} \times \mathbb{R}^{n} \rightarrow \mathbb{R}^{n}$ is continuous for each $\omega \in \Omega, \phi(t, \omega, x)$ is called a continuous random dynamical system. An RDS $\phi$ is called a smooth RDS of class $C^{k}, k \geq 1$, if for each $(t, \omega) \in \mathbb{R} \times \Omega, \phi(t, \omega, \cdot): \mathbb{R}^{n} \rightarrow \mathbb{R}^{n}$ is $C^{k}$-smooth. Namely, $\phi(t, \omega, x)$ is $k$ times differentiable in $x$ and the derivatives are continuous in $(t, x)$ for each $\omega \in \Omega$.

Random dynamical systems arise in the modeling of many phenomena in physics, biology, climatology, economics, etc. which are often subject to uncertainty or random influences, such as stochastic forcing, uncertain parameters, random sources or inputs, and random boundary conditions. An example is the solution operator for a random differential equation driven by a real noise:

$$
\frac{d x}{d t}=f\left(\theta_{t} \omega, x\right)
$$

where $x \in \mathbb{R}^{d}, f: \Omega \times \mathbb{R}^{d} \rightarrow \mathbb{R}^{d}$ is a measurable function and $f_{\omega}(t, \cdot) \equiv f\left(\theta_{t} \omega, \cdot\right) \in$ $L_{\text {loc }}\left(\mathbb{R}, C_{b}^{0,1}\right)$,

Here, $(\Omega, \mathcal{F}, \mathbb{P})$ is the classic Wiener space, i.e., $\Omega=\left\{\omega: \omega(\cdot) \in C\left(\mathbb{R}, \mathbb{R}^{d}\right), \omega(0)=\right.$ $0\}$ endowed with the open compact topology so that $\Omega$ is a Polish space and $\mathbb{P}$ is the Wiener measure. Define a measurable dynamical system $\theta^{t}$ on the probability space $(\Omega, \mathcal{F}, P)$ by the Wiener shift $\left(\theta^{t} \omega\right)(\cdot)=\omega(t+\cdot)-\omega(t)$ for $t>0$. It is well known that $P$ is invariant and ergodic under $\theta^{t}$. This measurable dynamical system $\theta^{t}$ is also called a metric dynamical system. This models the noise in the system; see $[\mathrm{A}$, page 60 , for details.

We consider a deterministic flow $\psi(t)(x) \equiv \psi(t, x)$ in $\mathbb{R}^{n}$ and its randomly perturbed flow (cocycle) $\phi(t, \omega)(x) \equiv \phi(t, \omega, x)$. Our main results can be summarized as

Theorem. Assume that $\psi(t)$ is a $C^{r}$ flow, $r \geq 1$, and has compact, connected $C^{r}$ normally hyperbolic invariant manifold $\mathcal{M} \subset \mathbb{R}^{n}$. Then there exists $\rho>0$ such that for any $C^{1}$ random flow $\phi(t, \omega)$ in $\mathbb{R}^{n}$, if

$$
\|\phi(t, \omega)-\psi(t)\|_{C^{1}}<\rho, \quad \text { for } t \in[0,1], \omega \in \Omega,
$$

then

(i) Persistence: $\phi(t, \omega)$ has a $C^{1}$ normally hyperbolic random invariant manifold $\tilde{\mathcal{M}}(\omega)$.

(ii) Smoothness: If $\phi(t, \omega)$ is $C^{r}$ and the normal hyperbolicity is sufficiently strong (see Theorem 2.1), then $\tilde{\mathcal{M}}(\omega)$ is a $C^{r}$ manifold diffeomorphic to $\mathcal{M}$ for each $\omega \in \Omega$.

(iii) Existence of stable manifolds: $\phi(t, \omega)$ has a stable manifold $\tilde{\mathcal{W}}^{s}(\omega)$ at $\tilde{\mathcal{M}}(\omega)$.

(iv) Existence of unstable manifolds: $\phi(t, \omega)$ has an unstable manifold $\tilde{\mathcal{W}}^{u}(\omega)$ at $\tilde{\mathcal{M}}(\omega)$.

(v) Persistence of overflowing and inflowing manifold: If $\psi(t)$ has an overflowing/inflowing manifold $\mathcal{M}$, then $\phi(t, \omega)$ has an overflowing/inflowing manifold $\tilde{\mathcal{M}}(\omega)$.

As was stated earlier, when the perturbed flow $\phi(t, \omega)$ is a deterministic flow, i.e., $\phi(t, \omega)$ is independent of $\omega$, this theorem was proved by Fenichel [F1], and we follow the approach given there. The basic idea is due to Hadamard [H] and involves a graph transform to first construct a center-unstable manifold. One takes a Lipschitz graph over the unstable bundle at the deterministic normally hyperbolic invariant manifold for $\psi(t)$ and maps it forward under $\phi(t, \omega)$ for large but fixed $t$ for each 
$\omega \in \Omega$. Because each $\phi(t, \omega)$ is $C^{1}$ close to $\psi(t)$ and because the latter stretches the graph in the unstable direction while compressing in the normal direction, the image (for each $\omega$ ) is again a Lipschitz graph. Furthermore, this mapping is a contraction on the space of such graphs and the resulting fixed graph (for each $\omega$ ) is the centerunstable manifold. The center-stable manifold is obtained in the same way, by reversing time. The desired manifold is then the intersection of these two (for each $\omega)$. There are important technical hurdles that must be overcome. The fact that the manifolds are global forces us to use local charts, which is a messy but harmless complication. The fact that we are dealing with a random dynamical system also leads to some complicated notation and bookkeeping. The main difficulty, however, is the need to show certain measurability properties, and this is the crux of the extension. Of course, a random dynamical system is also nonautonomous, and so naturally the concept of an invariant manifold must be extended. Here, one is faced with the need to construct random invariant manifolds that change with time according to a metric dynamical system on a probability space (see the next section for definitions).

It should be noted that there are other approaches to proving perturbation results for normally hyperbolic invariant manifolds, most notably the Lyapunov-Perron method, but we believe that the obstacles are equally challenging and so take this more geometric route.

We mentioned the fact that persistence of invariant manifolds under perturbation is the basis for geometric singular perturbation theory. This paper is to be followed with another which gives the next step, that of constructing random invariant foliations (the counterpart of the Fenichel fibration) of the center-stable and centerunstable manifolds for $\phi(t, \omega)$. We also expect to develop an exchange lemma (see [JK]) for random dynamical systems. We hope that these will lay the groundwork for applications and provide tools for studying stochastic systems.

At present we do not have results along the lines of this paper for infinitedimensional random semiflows, as one encounters with stochastic parabolic PDEs. The deterministic case was treated in [BLZ1, BLZ2, BLZ3, but the extension to random systems is elusive.

The results we present here are closely related to the theory of local random stable, unstable, and center manifolds of stationary solutions. For finite-dimensional random dynamical systems, we refer to $[\mathrm{C}, \mathrm{BK}, \mathrm{Bx}, \mathrm{D}, \mathrm{LQ}, \mathrm{S}, \mathrm{W}, \mathrm{A}, \mathrm{LiL}$. The results on local invariant manifolds for infinite-dimensional random dynamical systems can be found in [R, CLR, DSL1, DSL2, MS, MZZ, LS, GLS, CDLS, WD]. Random inertial manifolds were obtained in $\mathrm{BF}, \mathrm{GC}, \mathrm{DD}$. Included in the previously cited work are discrete time dynamics with products of diffeomorphisms, in Euclidean space, on Riemannian manifolds, or in infinite-dimensional spaces, where applications to PDEs are considered. Various approaches have been used, the Lyapunov-Perron method in particular.

\section{MAin RESUltS}

In this section, we first introduce some basic concepts on random dynamical systems. Then, we state our main results.

2.1. Random dynamical systems. In this subsection, we review some of the basic concepts related to random dynamical systems in a Banach space that are 
taken from Arnold $[\mathrm{A}$. Let $(\Omega, \mathcal{F}, P)$ be a probability space and $X$ be a Banach space. Let $\mathbb{T}=\mathbb{R}$ or $\mathbb{Z}$ endowed with their Borel $\sigma$-algebra.

Definition 2.1. A family $\left(\theta^{t}\right)_{t \in \mathbb{T}}$ of mappings from $\Omega$ into itself is called a metric dynamical system if

(1) $(\omega, t) \rightarrow \theta^{t} \omega$ is $\mathcal{F} \otimes \mathcal{B}(\mathbb{T})$ measurable;

(2) $\theta^{0}=i d_{\Omega}$, the identity on $\Omega, \theta^{t+s}=\theta^{t} \circ \theta^{s}$ for all $t, s \in \mathbb{T}$;

(3) $\theta^{t}$ preserves the probability measure $P$.

Definition 2.2. A map

$$
\phi: \mathbb{T} \times \Omega \times X \rightarrow X, \quad(t, \omega, x) \mapsto \phi(t, \omega, x),
$$

is called a random dynamical system (or a cocycle) on the Banach space $X$ over a metric dynamical system $\left(\Omega, \mathcal{F}, P, \theta^{t}\right)_{t \in \mathbb{T}}$ if

(1) $\phi$ is $\mathcal{B}(\mathbb{T}) \otimes \mathcal{F} \otimes \mathcal{B}(X)$-measurable;

(2) the mappings $\phi(t, \omega):=\phi(t, \omega, \cdot): X \rightarrow X$ form a cocycle over $\theta^{t}$ :

$$
\begin{aligned}
& \phi(0, \omega)=I d, \quad \text { for all } \omega \in \Omega, \\
& \phi(t+s, \omega)=\phi\left(t, \theta^{s} \omega\right) \circ \phi(s, \omega), \quad \text { for all } t, s \in \mathbb{T}, \quad \omega \in \Omega .
\end{aligned}
$$

An example is the solution operator for a random differential equation driven by a real noise,

$$
\frac{d x}{d t}=f\left(\theta_{t} \omega, x\right)
$$

where $x \in \mathbb{R}^{d}, f: \Omega \times \mathbb{R}^{d} \rightarrow \mathbb{R}^{d}$ is a measurable function and $f_{\omega}(t, \cdot) \equiv f\left(\theta_{t} \omega, \cdot\right) \in$ $L_{\text {loc }}\left(\mathbb{R}, C_{b}^{0,1}\right)$.

2.2. Normally hyperbolic random invariant manifolds. We first recall that a multifunction $\mathcal{M}=(\mathcal{M}(\omega))_{\omega \in \Omega}$ of nonempty closed sets $\mathcal{M}(\omega), \omega \in \Omega$, contained in a separable Banach space $X$ is called a random set if

$$
\omega \mapsto \inf _{y \in \mathcal{M}(\omega)}\|x-y\|
$$

is a random variable for any $x \in X$. When each of the sets $\mathcal{M}(\omega)$ is a manifold, we call $\mathcal{M}$ a random manifold.

Definition 2.3. A random manifold $\mathcal{M}$ is called a random invariant manifold for a random dynamical system $\phi(t, \omega)$ if

$$
\phi(t, \omega, \mathcal{M}(\omega))=\mathcal{M}\left(\theta^{t} \omega\right) \text { for all } t \in \mathbb{R}, \omega \in \Omega .
$$

Definition 2.4. A random invariant manifold $\mathcal{M}$ is said to be normally hyperbolic if for almost every $\omega \in \Omega$ and $x \in \mathcal{M}(\omega)$, there exists a splitting which is $C^{0}$ in $x$ and measurable in $\omega$ :

$$
X=E^{u}(\omega, x) \oplus E^{c}(\omega, x) \oplus E^{s}(\omega, x)
$$

of closed subspaces with associated projections $\Pi^{u}(\omega, x), \Pi^{c}(\omega, x)$, and $\Pi^{s}(\omega, x)$ such that

(i) The splitting is invariant:

$$
D_{x} \phi(t, \omega)(x) E^{i}(\omega, x)=E^{i}\left(\theta_{t} \omega, \phi(t, \omega)(x)\right), \quad \text { for } i=u, c,
$$

and

$$
D_{x} \phi(t, \omega)(x) E^{s}(\omega, x) \subset E^{s}\left(\theta_{t} \omega, \phi(t, \omega)(x)\right) .
$$


(ii) $\left.D_{x} \phi(t, \omega)(x)\right|_{E^{i}(\omega, x)}: E^{i}(\omega, x) \rightarrow E^{i}\left(\theta_{t} \omega, \phi(t, \omega)(x)\right)$ is an isomorphism for $i=u, c . E^{c}(\omega, x)$ is the tangent space of $\mathcal{M}(\omega)$ at $x$.

(iii) There are $(\theta, \phi)$-invariant random variables $\bar{\alpha}, \bar{\beta}: \mathcal{M} \rightarrow(0, \infty), \bar{\alpha}<\bar{\beta}$, and a tempered random variable $K: \mathcal{M} \rightarrow[1, \infty)$ such that

$$
\begin{aligned}
& \left\|D_{x} \phi(t, \omega)(x) \Pi_{s}(\omega, x)\right\| \leq K(\omega, x) e^{-\bar{\beta} \omega, x) t} \quad \text { for } t \geq 0, \\
& \left\|D_{x} \phi(t, \omega)(x) \Pi_{u}(\omega, x)\right\| \leq K(\omega, x) e^{\bar{\beta}(\omega, x) t} \quad \text { for } t \leq 0, \\
& \left\|D_{x} \phi(t, \omega)(x) \Pi_{c}(\omega, x)\right\| \leq K(\omega, x) e^{\bar{\alpha}(\omega, x)|t|} \quad \text { for }-\infty<t<\infty .
\end{aligned}
$$

We consider a deterministic flow $\psi(t)(x) \equiv \psi(t, x)$ in $\mathbb{R}^{n}$ and its randomly perturbed (cocycle) counterpart $\phi(t, \omega)(x) \equiv \phi(t, \omega, x)$. Our main results are:

Theorem 2.1. Assume that $\psi(t)$ is a $C^{r}$ flow, $r \geq 1$, and has a compact, connected $C^{r}$ normally hyperbolic invariant manifold $\mathcal{M} \subset \mathbb{R}^{n}$. Let the positive exponents related to the normal hyperbolicity be $\alpha<\beta$ in (2.1)-(2.3), which in this case are constant and deterministic. Then there exists $\rho>0$ such that for any random $C^{1}$ flow $\phi(t, \omega)$ in $\mathbb{R}^{n}$, if

$$
\|\phi(t, \omega)-\psi(t)\|_{C^{1}}<\rho, \quad \text { for } t \in[0,1], \omega \in \Omega,
$$

then

(i) Persistence: $\phi(t, \omega)$ has a $C^{1}$ normally hyperbolic random invariant manifold $\tilde{\mathcal{M}}(\omega)$.

(ii) Smoothness: If $\alpha<r \beta$ and $\phi(t, \omega)$ is $C^{r}$, then $\tilde{\mathcal{M}}(\omega)$ is a $C^{r}$ manifold diffeomorphic to $\mathcal{M}$ for each $\omega \in \Omega$.

(iii) Existence of stable manifolds: $\phi(t, \omega)$ has a stable manifold $\tilde{\mathcal{W}}^{s}(\omega)$ at $\tilde{\mathcal{M}}(\omega)$.

(iv) Existence of unstable manifolds: $\phi(t, \omega)$ has an unstable manifold $\tilde{\mathcal{W}}^{u}(\omega)$ at $\tilde{\mathcal{M}}(\omega)$.

We use the notation $B(0, r)$ to refer to the ball centered at 0 of radius $r$ where the space is clear from the context. We will also use the notation $L \mid E$ to mean the restriction of the linear operator $L$ to the subspace $E$. We do not place $E$ as a subscript, since often it has sub and superscripts.

\subsection{Normally hyperbolic random overflowing and inflowing invariant manifolds.}

Definition 2.5. A random manifold $\overline{\mathcal{M}}(\omega)=\mathcal{M}(\omega) \cup \partial \mathcal{M}(\omega)$ is called a random overflowing invariant manifold for a random dynamical system $\phi(t, \omega)$ if

$$
\phi(t, \omega, \mathcal{M}(\omega)) \supset \overline{\mathcal{M}}\left(\theta^{t} \omega\right) \text { for all } t>0, \omega \in \Omega .
$$

Definition 2.6. A random manifold $\overline{\mathcal{M}}(\omega)=\mathcal{M}(\omega) \cup \partial \mathcal{M}(\omega)$ is called a random inflowing invariant manifold for a random dynamical system $\phi(t, \omega)$ if

$$
\phi(t, \omega, \mathcal{M}(\omega)) \supset \overline{\mathcal{M}}\left(\theta^{t} \omega\right) \text { for all } t<0, \omega \in \Omega .
$$

Definition 2.7. A random overflowing invariant manifold $\overline{\mathcal{M}}(\omega)=\mathcal{M}(\omega) \cup \partial \mathcal{M}(\omega)$ is said to be normally (stably) hyperbolic if for almost every $\omega \in \Omega$ and $x \in \mathcal{M}(\omega)$, there exists a splitting which is $C^{0}$ in $x$ and measurable in $\omega$ :

$$
X=E^{c}(\omega, x) \oplus E^{s}(\omega, x)
$$


of closed subspaces with associated projections $\Pi^{c}(\omega, x)$ and $\Pi^{s}(\omega, x)$ such that

(i) The splitting is invariant:

$$
D \phi\left(t, \theta^{-t} \omega\right)(\phi(-t, \omega)(x)) E^{c}\left(\theta^{-t} \omega, \phi(-t, \omega)(x)\right)=E^{c}(\omega, x)
$$

and

$$
D \phi\left(t, \theta^{-t} \omega\right)(\phi(-t, \omega)(x)) E^{s}\left(\theta^{-t} \omega, \phi(-t, \omega)(x)\right) \subset E^{s}(\omega, x) .
$$

(ii) $D \phi\left(t, \theta^{-t} \omega\right)(\phi(-t, \omega)(x)) \mid E^{c}\left(\theta^{-t} \omega, \phi(-t, \omega)(x)\right): E^{c}\left(\theta^{-t} \omega, \phi(-t, \omega)(x)\right) \rightarrow$ $E^{c}(\omega, x)$ is an isomorphism. $E^{c}(\omega, x)$ is the tangent space of $\mathcal{M}(\omega)$ at $x$.

(iii) There are $(\theta, \phi)$-invariant random variables $\alpha, \beta: \mathcal{M} \rightarrow(0, \infty), \alpha<\beta$, and a tempered random variable $K(\omega, x): \mathcal{M} \rightarrow[1, \infty)$ such that

$$
\left\|D \phi\left(t, \theta^{-t} \omega\right)(\phi(-t, \omega)(x)) \Pi_{s}\right\| \leq K\left(\theta^{-t} \omega, \phi(-t, \omega)(x)\right) e^{-\beta(\omega, x) t} \quad \text { for } t \geq 0
$$

$$
\left\|D_{x} \phi(t, \omega)(x) \Pi_{c}(\omega, x)\right\| \leq K(\omega, x) e^{\alpha(\omega, x)|t|} \quad \text { for } t \text { such that } \phi(t, \omega, x) \in \mathcal{M}\left(\theta^{t} \omega\right) .
$$

Definition 2.8. A random inflowing invariant manifold $\overline{\mathcal{M}}(\omega)=\mathcal{M}(\omega) \cup \partial \mathcal{M}(\omega)$ is said to be normally (unstably) hyperbolic if for almost every $\omega \in \Omega$ and $x \in \mathcal{M}(\omega)$, there exists a splitting which is $C^{0}$ in $x$ and measurable in $\omega$ :

$$
X=E^{u}(\omega, x) \oplus E^{c}(\omega, x)
$$

of closed subspaces with associated projections $\Pi^{u}(\omega, x)$ and $\Pi^{c}(\omega, x)$ such that

(i) The splitting is invariant:

$$
\left.D \phi(t, \omega, x) E^{i}(\omega, x)\right)=E^{i}\left(\theta^{t} \omega, \phi(t, \omega)(x), \quad \text { for } i=u, c .\right.
$$

(ii) $D \phi\left(t, \theta^{-t} \omega\right)(\phi(-t, \omega)(x)) \mid E^{i}\left(\theta^{-t} \omega, \phi(-t, \omega)(x)\right): E^{i}\left(\theta^{-t} \omega, \phi(-t, \omega)(x)\right) \rightarrow$ $E^{i}(\omega, x)$ is an isomorphism for $i=u, c . E^{c}(\omega, x)$ is the tangent space of $\mathcal{M}(\omega)$ at $x$.

(iii) There are $(\theta, \phi)$-invariant random variables $\bar{\alpha}, \bar{\beta}: \mathcal{M} \rightarrow(0, \infty), \bar{\alpha}<\bar{\beta}$, and a tempered random variable $K(\omega, x): \mathcal{M} \rightarrow[1, \infty)$ such that

$$
\left\|D \phi\left(t, \theta^{-t} \omega\right)(\phi(-t, \omega, x)) \Pi_{u}\right\| \leq K\left(\theta^{-t} \omega, \phi(-t, \omega, x)\right) e^{\bar{\beta}(\omega, x) t} \quad \text { for } t \leq 0,
$$

$$
\left\|D_{x} \phi(t, \omega)(x) \Pi_{c}(\omega, x)\right\| \leq K(\omega, x) e^{\bar{\alpha}(\omega, x)|t|} \quad \text { for } t \text { such that } \phi(t, \omega, x) \in \mathcal{M}\left(\theta^{t} \omega\right) \text {. }
$$

Theorem 2.2. Assume that $\psi(t)$ is a $C^{r}$ flow, $r \geq 1$, and has a compact, connected $C^{r}$ normally hyperbolic overflowing invariant manifold $\overline{\mathcal{M}}=\mathcal{M} \cup \partial \mathcal{M} \subset \mathbb{R}^{n}$ with positive deterministic constant hyperbolicity exponents $\alpha<\beta$. Then there exists $\rho>0$ such that for any random $C^{r}$ flow $\phi(t, \omega)$ in $\mathbb{R}^{n}$, if

$$
\|\phi(t, \omega)-\psi(t)\|_{C^{1}}<\rho, \quad \text { for } t \in[0,1], \omega \in \Omega,
$$

and if $\alpha<r \beta$, then $\phi(t, \omega)$ has a $C^{r}$ normally hyperbolic random overflowing invariant manifold $\tilde{\mathcal{M}}(\omega)$ such that for each $\omega \in \Omega, \tilde{\mathcal{M}}(\omega)$ is $C^{r}$ diffeomorphic to $\mathcal{M}$.

Theorem 2.3. Assume that $\psi(t)$ is a $C^{r}$ flow, $r \geq 1$, and has a compact, connected $C^{r}$ normally hyperbolic inflowing invariant manifold $\overline{\mathcal{M}}=\mathcal{M} \cup \partial \mathcal{M} \subset \mathbb{R}^{n}$. Then there exists $\rho>0$ such that for any random $C^{r}$ flow $\phi(t, \omega)$ in $\mathbb{R}^{n}$, if

$$
\|\phi(t, \omega)-\psi(t)\|_{C^{1}}<\rho, \quad \text { for } t \in[0,1], \omega \in \Omega,
$$

and if $\alpha<r \beta$, then $\phi(t, \omega)$ has a $C^{r}$ normally hyperbolic random inflowing invariant manifold $\tilde{\mathcal{M}}(\omega)$ such that for each $\omega \in \Omega, \tilde{\mathcal{M}}(\omega)$ is $C^{r}$ diffeomorphic to $\mathcal{M}$. 


\section{BASIC LEMMAS}

In this section, we first recall some facts for deterministic system taken from [F1. Then we deduce the corresponding properties for random systems.

We consider a compact connected $C^{r}$ normally hyperbolic invariant manifold $\mathcal{M}$ under the deterministic $C^{r}$ flow $\psi(t)$ and let $T \mathbb{R}^{n} \mid \mathcal{M}=T \mathcal{M} \oplus E^{s} \oplus E^{u}$ be the associated splitting. We will sometimes use the notation $E^{c}$ to denote the bundle $T \mathcal{M}$, and we will sometimes use $E^{i}$ to denote the subspace $E_{m}^{i}(i=c, s, u)$ at a point $m \in \mathcal{M}$ when the context makes the meaning obvious. We denote the associated positive constant hyperbolicity exponents by $\alpha<\beta$.

The first two propositions concern the hyperbolicity of the invariant manifold $\mathcal{M}$.

Proposition 3.1. Normal hyperbolicity is independent of the metric.

Proposition 3.2. If $\mathcal{M}$ is normally hyperbolic under the flow $\psi(t)$ :

(1) There exist positive constants $a<1$ and $c_{1}$ such that

$$
\left\|D \psi(t)(\psi(-t)(m)) \mid E^{s}\right\|<c_{1} a^{t}
$$

for all $m \in \mathcal{M}$ and $t \geq 0$ and

$$
\left\|D \psi(t)(\psi(-t)(m)) \mid E^{u}\right\|<c_{1} a^{-t}
$$

for all $m \in \mathcal{M}$ and $t \leq 0$.

(2) If $\alpha<r \beta$, there exist $c_{2}>0$ and $r^{\prime}>r$ such that

$$
\left\|D \psi(t)(\psi(-t)(m))\left|E^{s}\|\| D(\psi(-t))(m)\right| E^{c}\right\|^{r^{\prime}}<c_{2}
$$

for all $m \in \mathcal{M}$ and $t \geq 0$ and

$$
\left\|D \psi(t)(\psi(-t)(m))\left|E^{u}\|\| D(\psi(-t))(m)\right| E^{c}\right\|^{r^{\prime}}<c_{2}
$$

for all $m \in \mathcal{M}$ and $t \leq 0$.

Let the dimensions of $E^{s}, E^{u}$, and $\mathcal{M}$ be $k, l, m$, respectively, so that $k+l+m=$ $n$, and let $\Pi^{s}, \Pi^{u}, \Pi^{c}$ denote the projections corresponding to the splitting. The next two propositions are on the smooth approximation of normal bundles and the tubular neighborhood of $\mathcal{M}$; see [W].

Proposition 3.3. There exist $k$ - and l-dimensional $C^{r}$ subbundles $\tilde{E}^{s}$ and $\tilde{E}^{u}$ of $T \mathbb{R}^{n} \mid \mathcal{M}$ which are arbitrarily close to $E^{s}$ and $E^{u}$, respectively, and such that $T \mathcal{M} \oplus \tilde{E}^{i}$ is invariant under $D \psi(t)$, i.e., $T \mathcal{M} \oplus \tilde{E}^{i}=T \mathcal{M} \oplus E^{i}$ for $i=s, u$.

We have $T \mathbb{R}^{n} \mid \mathcal{M}=\tilde{E}^{s} \oplus \tilde{E}^{u} \oplus T \mathcal{M}$ and denote by $\tilde{\Pi}^{s}, \tilde{\Pi}^{u}$ and $\tilde{\Pi}^{c}$ the projections corresponding to the splitting. We sometimes use the notation $\tilde{E}=\tilde{E}^{s} \oplus \tilde{E}^{u}$.

Proposition 3.4. There exists a $C^{r}$ diffeomorphism $\gamma$ from a neighborhood of the zero section of $\tilde{E}$ to a tubular neighborhood of $\mathcal{M}$ in $\mathbb{R}^{n}$.

To construct the random unstable and stable manifolds, we first introduce the local coordinate charts in $\mathbb{R}^{n}$ near $\mathcal{M}$.

We use the normal bundle $\tilde{E}$ to define local charts in $\mathbb{R}^{n}$ near $\mathcal{M}$ as in [F1. By compactness of $\mathcal{M}$, we can take finitely many points $p_{1}, p_{2}, \cdots, p_{s}$ of $\mathcal{M}$ with the following properties: for each $p_{j}$, there exists a coordinate neighborhood $U^{j}$ of $p_{j}$ in $\mathcal{M} ;\left\{U^{j}\right\}$ covers $\mathcal{M}$; in each $U^{j}, \tilde{E}$ has an orthonormal basis which changes in a $C^{r}$ manner. Moreover, we can shrink each $U^{j}$ a little to $\tilde{U}^{j}$ such that $\overline{\tilde{U}^{j}} \subset U^{j}$ 
and $\left\{\tilde{U}^{j}\right\}$ still cover $\mathcal{M}$. Suppose $\left\{\left(\sigma_{j}, U_{5}^{j}\left(=U^{j}\right)\right)\right\}$ is an atlas on $\mathcal{M}$ and $\sigma\left(U_{5}^{j}\right)=$ $\mathcal{D}_{5}^{j} \subset \mathbb{R}^{m}$ is an open neighborhood of the origin in $\mathbb{R}^{m}$. We may take open subsets $\mathcal{D}_{1}^{j}, \mathcal{D}_{2}^{j}, \mathcal{D}_{3}^{j}, \mathcal{D}_{4}^{j}$ of $\mathcal{D}_{5}^{j}$ such that $\overline{\mathcal{D}_{i-1}^{j}} \subset \mathcal{D}_{i}^{j}$ for $i=2,3,4,5$ and $\overline{\tilde{U}^{j}} \subset \sigma_{j}^{-1}\left(\mathcal{D}_{1}^{j}\right)$. Taking $U_{i}^{j}=\sigma_{j}^{-1}\left(\mathcal{D}_{i}^{j}\right), U_{i}^{j}$ is an open neighborhood of $p_{j}$ and $\tilde{U}^{j} \subset U_{i}^{j}$ for all $i=1,2,3,4,5$. So $\left\{U_{i}^{j}\right\}_{j}$ cover $\mathcal{M}$. In fact, we have

$$
\mathcal{M}=\bigcup_{j} U_{1}^{j} \subset \bigcup_{j} U_{2}^{j} \subset \cdots \subset \bigcup_{j} U_{5}^{j}=\mathcal{M} .
$$

Define for small $\epsilon>0$

$$
\tilde{E}_{\epsilon}=\tilde{E}_{\epsilon}^{s} \oplus \tilde{E}_{\epsilon}^{u}:=\left\{\left(m, \nu^{s}, \nu^{u}\right) \in \tilde{E}:\left\|\nu^{s}\right\|<\epsilon,\left\|\nu^{u}\right\|<\epsilon\right\} .
$$

It follows from Proposition 3.4 that there exists $\epsilon_{0}>0$ such that for $0<\epsilon<\epsilon_{0}$, $\tilde{E}_{\epsilon}$ is $C^{r}$ diffeomorphic to a neighborhood $V$ of $\mathcal{M}$ in $\mathbb{R}^{n}$. In other words, $\tilde{E}_{\epsilon}$ is a tubular neighborhood of $\mathcal{M}$. From now on, we view both $\psi(t, x)$ and $\phi(t, \omega, x)$ as flows on $\tilde{E}_{\epsilon}$ as well as on $V$.

Choose a $C^{r}$ orthonormal basis on $\tilde{E} \mid U_{5}^{j}$ for each $j$. Thus, for $m \in U_{5}^{j}$ and $\left(m, \nu^{s}\right) \in \tilde{E}_{\epsilon}^{s}, \nu^{s}$ has coordinates $\left(x_{1}^{s}, \cdots, x_{k}^{s}\right)$ under the chosen orthonormal basis, and these depend smoothly on $m$. Define $\tau_{j}^{s}: \tilde{E}_{\epsilon}^{s} \mid U_{5}^{j} \rightarrow \mathbb{R}^{k}$ by

$$
\tau_{j}^{s}\left(m, \nu^{s}\right)=x^{s}=\left(x_{1}^{s}, \cdots, x_{k}^{s}\right) \in \mathbb{R}^{k}
$$

and define $\tau_{j}^{u}$ similarly by

$$
\tau_{j}^{u}\left(m, \nu^{u}\right)=x^{u}=\left(x_{1}^{u}, \cdots, x_{l}^{u}\right) \in \mathbb{R}^{l} .
$$

We have

$$
\left\|x^{s}\right\|_{\mathbb{R}^{k}}=\left\|\nu^{s}\right\|,\left\|x^{u}\right\|_{\mathbb{R}^{l}}=\left\|\nu^{u}\right\| .
$$

These give us $C^{r}$ diffeomorphisms $\gamma_{j}$ from $\tilde{E}_{\epsilon} \mid U_{5}^{j}$ to $\mathbb{R}^{n}=\mathbb{R}^{m} \oplus \mathbb{R}^{k} \oplus \mathbb{R}^{l}$, where

$$
\gamma_{j}\left(m, \nu^{s}, \nu^{u}\right)=\left(x^{c}, x^{s}, x^{u}\right)=\left(\sigma_{j}(m), \tau_{j}^{s}\left(m, \nu^{s}\right), \tau_{j}^{u}\left(m, \nu^{u}\right)\right) .
$$

So $\left\{\left(\tilde{E}_{\epsilon} \mid U_{5}^{j}, \gamma_{j}\right)\right\}$ gives us an atlas on $\tilde{E}_{\epsilon}$.

Since $\mathcal{M}$ is compact, there exists $L_{1}>0$ such that $D \sigma_{j}$ and $D \sigma_{j}^{-1}$ are bounded by $L_{1}$ for all $m$ and $j$.

Then for sufficiently large $T>0$, we represent the random diffeomorphism $\phi(T, \omega)(\cdot)$ in the local charts by

$$
\left(x^{c}, x^{s}, x^{u}\right) \rightarrow\left(f_{i j}^{c}\left(\omega, x^{c}, x^{s}, x^{u}\right), f_{i j}^{s}\left(\omega, x^{c}, x^{s}, x^{u}\right), f_{i j}^{u}\left(\omega, x^{c}, x^{s}, x^{u}\right)\right),
$$

where

$$
f_{i j}^{l}\left(\omega, x^{c}, x^{s}, x^{u}\right)=P_{l} \circ \gamma_{j} \circ \phi(T, \omega, \cdot) \circ \gamma_{i}^{-1}\left(x^{c}, x^{s}, x^{u}\right)
$$

for $\left(x^{c}, x^{s}, x^{u}\right) \in \gamma_{i} \circ \tilde{E}_{\epsilon} \mid \overline{U_{4}^{i} \cap \psi\left(-T, U_{4}^{j}\right)}$ and $P_{l}\left(x^{c}, x^{s}, x^{u}\right)=x^{l}$ for $l=c, s, u$. Note that given $i$ and $\left(x^{c}, x^{s}, x^{u}\right), f_{i j}\left(\omega, x^{c}, x^{s}, x^{u}\right)$ is defined only for some $j$ 's.

Denoting the differential operators $D_{1}=D_{x^{c}}, D_{2}=D_{x^{s}}, D_{3}=D_{x^{u}}$, we have

$$
\begin{aligned}
& D_{2} f_{i j}^{s}\left(\omega, x^{c}, x^{s}, x^{u}\right) \\
= & D P_{l} D \gamma_{j} D \phi(T, \omega) D_{2} \gamma^{-1}\left(x^{c}, x^{s}, x^{u}\right) \\
= & D P_{l} D \gamma_{j}\left(\tilde{\Pi}^{s} D \phi(T, \omega) \mid \tilde{E}^{s}\right) D_{2} \gamma_{i}^{-1}\left(x^{c}, x^{s}, x^{u}\right) .
\end{aligned}
$$

Since $\mathcal{M}$ is compact, $D P_{l}, D \gamma_{j}$, and $D_{2} \gamma^{-1}$, along with their inverses, are uniformly bounded by a constant $L_{2}>0$. Then

$$
\left\|D_{2} f_{i j}^{s}\left(\omega, x^{c}, x^{s}, x^{u}\right)\right\| \leq L_{2}^{3}\left\|\tilde{\Pi}^{s} D \phi(T, \omega) \mid \tilde{E}^{s}\right\| .
$$


Similarly, we get for $k=1,2,3$ and $l=c, s, u$,

$$
\left\|\left(D_{k} f_{i j}^{l}\left(\omega, x^{c}, x^{s}, x^{u}\right)\right)^{ \pm 1}\right\| \leq L\left\|\tilde{\Pi}^{l} D \phi(T, \omega) \mid \tilde{E}^{l}\right\|,
$$

for some $L>0$. We have the following lemma:

Lemma 3.1. There exists a $T>0$ such that for any $\eta>0$, if $\epsilon$ and $\rho$ are small enough, then

$$
\begin{gathered}
\left\|\left(D_{3} f_{i j}^{u}\left(\omega, x^{c}, x^{s}, x^{u}\right)\right)^{-1}\right\|<\frac{1}{4}, \\
\left\|D_{2} f_{i j}^{s}\left(\omega, x^{c}, x^{s}, x^{u}\right)\right\|<\frac{1}{2}, \\
\left\|\left(D_{1} f_{i j}^{c}\left(\omega, x^{c}, x^{s}, x^{u}\right)\right)^{-1}\right\|^{k}\left\|D_{2} f_{i j}^{s}\left(\omega, x^{c^{\prime}}, x^{s^{\prime}}, x^{u \prime}\right)\right\|<\frac{1}{4},
\end{gathered}
$$

for $0 \leq k \leq r, x^{c \prime}$ close to $x^{c}$, and

$$
\begin{gathered}
\left\|\left(D_{3} f_{i j}^{c}\left(\omega, x^{c}, x^{s}, x^{u}\right)\right)^{-1}\right\|<\eta, \\
\left\|f_{i j}^{s}\left(\omega, x^{c}, x^{s}, x^{u}\right)\right\|<\eta,\left\|f_{i j}^{u}\left(\omega, x^{c}, x^{s}, x^{u}\right)\right\|<\eta, \\
\left\|D_{1} f_{i j}^{s}\left(\omega, x^{c}, x^{s}, x^{u}\right)\right\|<\eta,\left\|D_{3} f_{i j}^{s}\left(\omega, x^{c}, x^{s}, x^{u}\right)\right\|<\eta, \\
\left\|D_{1} f_{i j}^{u}\left(\omega, x^{c}, x^{s}, x^{u}\right)\right\|<\eta,\left\|D_{2} f_{i j}^{u}\left(\omega, x^{c}, x^{s}, x^{u}\right)\right\|<\eta .
\end{gathered}
$$

Moreover, the norm of all first partial derivatives of $f_{i j}$ and their inverses are bounded by some $Q>0$.

Proof. By Proposition 3.2, there exists a $T>0$ such that

$$
\begin{gathered}
L^{r}\left\|D \psi(-T)(m) \mid E^{u}\right\|<\frac{1}{16}, \\
L^{r}\left\|D \psi(T)(\psi(-T)(m)) \mid E^{s}\right\|<\frac{1}{8}, \\
L^{r+1}\left\|D \psi(-T)(m)\left|T \mathcal{M}\left\|^{k}\right\| D \psi(T)(\psi(-T, m))\right| E^{s}\right\|<\frac{1}{16}, 0 \leq k \leq r .
\end{gathered}
$$

Since $\tilde{E}$ and $E$ are arbitrarily close, and $\|\phi(t, \omega)-\psi(t)\|_{C^{1}}<\rho$ for all $t \in[0,1]$ and $\omega \in \Omega$, if $\rho$ is small enough, we have

$$
L^{r+1}|| D \phi(-T, \omega)(m)\left|T \mathcal{M}\left\|^{k}|| \tilde{\Pi}^{s} D \phi(T, \omega)(\psi(-T)(m)) \mid \tilde{E}^{s}\right\|<\frac{1}{8}, 0 \leq k \leq r .\right.
$$

Note that we identified $V$ with $E(\epsilon)$ and viewed $\psi(t)$ and $\phi(t, \omega)$ as flows in the bundles. So in terms of $f_{i j}^{l}$,

$$
\begin{gathered}
\left\|\left(D_{3} f_{i j}^{u}\left(\omega, x^{c}, 0,0\right)\right)^{-1}\right\|<\frac{1}{8}, \\
\left\|D_{2} f_{i j}^{s}\left(\omega, x^{c}, 0,0\right)\right\|<\frac{1}{4},
\end{gathered}
$$




$$
\left\|\left(D_{1} f_{i j}^{c}\left(\omega, x^{c}, 0,0\right)\right)^{-1}\right\|^{k}\left\|D_{2} f_{i j}^{s}\left(\omega, x^{c}, 0,0\right)\right\|<\frac{1}{8}, 0 \leq k \leq r .
$$

We also have

$$
\left\|\left(D_{3} f_{i j}^{c}\left(\omega, x^{c}, 0,0\right)\right)^{-1}\right\|<\frac{1}{2} \eta .
$$

Choose $\epsilon$ and $\rho$ small enough, we have

$$
\begin{gathered}
\left\|\left(D_{3} f_{i j}^{u}\left(\omega, x^{c}, x^{s}, x^{u}\right)\right)^{-1}\right\|<\frac{1}{4}, \\
\left\|D_{2} f_{i j}^{s}\left(\omega, x^{c}, x^{s}, x^{u}\right)\right\|<\frac{1}{2}, \\
\left\|\left(D_{1} f_{i j}^{c}\left(\omega, x^{c}, x^{s}, x^{u}\right)\right)^{-1}\right\|^{k}\left\|D_{2} f_{i j}^{s}\left(\omega, x^{c^{\prime}}, x^{s^{\prime}}, x^{u \prime}\right)\right\|<\frac{1}{4},
\end{gathered}
$$

for $0 \leq k \leq r, x^{c \prime}$ close to $x^{c}$, and

$$
\left\|\left(D_{3} f_{i j}^{c}\left(\omega, x^{c}, x^{s}, x^{u}\right)\right)^{-1}\right\|<\eta .
$$

By the invariance of $\mathcal{M}$ under $\psi(T)$, the invariance of $E^{u}, E^{s}, T \mathcal{M}$ under $D \psi(T)$ and the closeness of $\tilde{E}$ and $E$, if $\epsilon$ and $\rho$ are small enough, we have

$$
\begin{gathered}
\left\|f_{i j}^{s}\left(\omega, x^{c}, x^{s}, x^{u}\right)\right\|<\eta,\left\|f_{i j}^{u}\left(\omega, x^{c}, x^{s}, x^{u}\right)\right\|<\eta, \\
\left\|D_{1} f_{i j}^{s}\left(\omega, x^{c}, x^{s}, x^{u}\right)\right\|<\eta,\left\|D_{3} f_{i j}^{s}\left(\omega, x^{c}, x^{s}, x^{u}\right)\right\|<\eta, \\
\left\|D_{1} f_{i j}^{u}\left(\omega, x^{c}, x^{s}, x^{u}\right)\right\|<\eta,\left\|D_{2} f_{i j}^{u}\left(\omega, x^{c}, x^{s}, x^{u}\right)\right\|<\eta .
\end{gathered}
$$

This completes the proof of the lemma.

\section{Existence of the RANDOM UnStable MANifold}

In this section, we will prove the existence of a random unstable manifold using the graph transform. The random unstable manifold will be constructed as a section of $\tilde{E}_{\epsilon}$ over $\tilde{E}_{\epsilon}^{u}$. We will define a transform on the space of all Lipschitz sections and prove that the transform has a fixed point which gives the random unstable manifold.

Let $X$ denote the space of sections of $\tilde{E}_{\epsilon}$ over $\tilde{E}_{\epsilon}^{u}$. For $u(\omega) \in X$, it is locally represented by $u_{i}(\omega)$ :

$$
u_{i}\left(\omega, x^{c}, x^{u}\right)=\tau_{i}^{s} \circ P^{s} \circ u(\omega) \circ\left(\sigma_{i} \times \tau_{i}^{u}\right)^{-1}\left(x^{c}, x^{u}\right),
$$

where $P^{s}$ is the fiber projection. Let $\epsilon>0$ be fixed so that $\bar{B}_{\epsilon}(0) \subset \mathcal{D}_{5}^{i}$ for all $j$. Define

$$
\begin{aligned}
\operatorname{Lip} u(\omega):= & \max _{i} \sup _{x^{c}, x^{c \prime} \in \mathcal{D}_{3}^{i},\left\|x^{u}\right\|,\left\|x^{u \prime}\right\| \leq \epsilon,\left(x^{c}, x^{u}\right) \neq\left(x^{c \prime}, x^{u \prime}\right)} \\
& \times \frac{\left\|u_{i}\left(\omega, x^{c}, x^{u}\right)-u_{i}\left(\omega, x^{c \prime}, x^{u \prime}\right)\right\|}{\max \left\{\left\|x^{c}-x^{c \prime}\right\|,\left\|x^{u}-x^{u \prime}\right\|\right\}}
\end{aligned}
$$

if it exists. Denote $X_{\delta}=\{u(\omega) \in X \mid \operatorname{Lip} u(\omega) \leq \delta\}$. Denote a random section by

$$
u=\{u(\omega) \in X \mid \omega \in \Omega\}
$$

and denote by $S$ the set of all such $u=\{u(\omega): \omega \in \Omega\}$. Define

$$
\text { Lip } u:=\sup _{\omega} \operatorname{Lip} u(\omega)
$$

and let

$$
S_{\delta}=\{u \in S \mid \operatorname{Lip} u \leq \delta\}
$$


Define the norm on $S_{\delta}$ by

$$
\|u\|:=\sup _{\omega} \max _{i} \sup _{x^{c}, x^{u}}\left|u_{i}\left(\omega, x^{c}, x^{u}\right)\right| .
$$

Then the induced metric on $S_{\delta}$ makes it into a complete metric space. Let $T$ be given by Lemma 3.1 .

Proposition 4.1. There is a unique $u \in S$ such that

$$
\phi(t, \omega, \operatorname{graph} u(\omega)) \supset \operatorname{graph} u\left(\theta^{t} \omega\right)
$$

for all $t>T$ and $\omega \in \Omega$. Furthermore, $u \in S_{\delta}$.

We need several lemmas to prove this proposition. We occasionaly write $u(\omega)$ in place of graph $u(\omega)$.

In the local coordinates, the mapping $u(\omega) \rightarrow \phi(T, \omega)(u(\omega))$ has the form

$$
\begin{aligned}
\left(x^{c}, u_{i}\left(\omega, x^{c}, x^{u}\right), x^{u}\right) \rightarrow & \left(f_{i j}^{c}\left(\omega, x^{c}, u_{i}\left(\omega, x^{c}, x^{u}\right), x^{u}\right), f_{i j}^{s}\left(\omega, x^{c}, u_{i}\left(\omega, x^{c}, x^{u}\right), x^{u}\right),\right. \\
& \left.f_{i j}^{u}\left(\omega, x^{c}, u_{i}\left(\omega, x^{c}, x^{u}\right), x^{u}\right)\right) .
\end{aligned}
$$

Define an operator $G$ on $S_{\delta}$ by

$$
(G u)_{j}\left(\omega, \xi^{c}, \xi^{u}\right)=f_{i j}^{s}\left(\theta^{-T} \omega, x^{c}, u_{i}\left(\theta^{-T} \omega, x^{c}, x^{u}\right), x^{u}\right),
$$

where

$$
\begin{aligned}
& \xi^{c}=f_{i j}^{c}\left(\theta^{-T} \omega, x^{c}, u_{i}\left(\theta^{-T} \omega, x^{c}, x^{u}\right), x^{u}\right), \\
& \xi^{u}=f_{i j}^{u}\left(\theta^{-T} \omega, x^{c}, u_{i}\left(\theta^{-T} \omega, x^{c}, x^{u}\right), x^{u}\right) .
\end{aligned}
$$

In the following, we will show $G$ is well defined. For convenience, we denote $x^{c u}=\left(x^{c}, x^{u}\right), \xi^{c u}=\left(\xi^{c}, \xi^{u}\right)$, and write

$$
f_{i j}^{s}\left(\omega, x^{c u}, x^{s}\right)=f_{i j}^{s}\left(\omega, x^{c}, x^{s}, x^{u}\right)
$$

and

$$
f_{i j}^{c u}\left(\omega, x^{c u}, x^{s}\right)=\left(f_{i j}^{c}\left(\omega, x^{c}, x^{s}, x^{u}\right), f_{i j}^{u}\left(\omega, x^{c}, x^{s}, x^{u}\right)\right)^{T} .
$$

We also use the norms

$$
\begin{aligned}
\left\|x^{c u}\right\| & =\max \left(\left\|x^{c}\right\|,\left\|x^{u}\right\|\right), \\
\left\|\xi^{c u}\right\| & =\max \left(\left\|\xi^{c}\right\|,\left\|\xi^{u}\right\|\right), \\
\left\|f_{i j}^{c u}\right\| & =\max \left(\left\|f_{i j}^{c}\right\|,\left\|f_{i j}^{u}\right\|\right) .
\end{aligned}
$$

Thus, we may write the local representatives as

$$
\begin{gathered}
u_{i}\left(\omega, x^{c u}\right)=u_{i}\left(\omega, x^{c}, x^{u}\right), \\
(G u)_{j}\left(\xi^{c u}\right)=f_{i j}^{s}\left(\omega, x^{c u}, u\left(\omega, x^{c u}\right)\right)
\end{gathered}
$$

with

Denote

$$
\xi^{c u}=f_{i j}^{c u}\left(\omega, x^{c u}, u\left(\omega, x^{c u}\right)\right)
$$

$$
\begin{aligned}
& A \equiv D_{1,3} f_{i j}^{s}\left(\omega, x^{c u}, x^{s}\right) \equiv\left(D_{1} f_{i j}^{s}\left(\omega, x^{c}, x^{s}, x^{u}\right), D_{3} f_{i j}^{s}\left(\omega, x^{c}, x^{s}, x^{u}\right)\right), \\
& B \equiv D_{2} f_{i j}^{s}\left(\omega, x^{c u}, x^{s}\right)=D_{2} f_{i j}^{s}\left(\omega, x^{c}, x^{s}, x^{u}\right), \\
& C \equiv D_{1,3} f_{i j}^{c u}\left(\omega, x^{c u}, x^{s}\right)=\left(\begin{array}{cc}
D_{1} f_{i j}^{c}\left(\omega, x^{c}, x^{s}, x^{u}\right) & D_{3} f_{i j}^{c}\left(\omega, x^{c}, x^{s}, x^{u}\right) \\
D_{1} f_{i j}^{u}\left(\omega, x^{c}, x^{s}, x^{u}\right) & D_{3} f_{i j}^{u}\left(\omega, x^{c}, x^{s}, x^{u}\right)
\end{array}\right),
\end{aligned}
$$

and

$$
E \equiv D_{2} f_{i j}^{c u}\left(\omega, x^{c u}, x^{s}\right)=\left(D_{2} f_{i j}^{c}\left(\omega, x^{c}, x^{s}, x^{u}\right), D_{2} f_{i j}^{u}\left(\omega, x^{c}, x^{s}, x^{u}\right)\right) .
$$


Lemma 4.1. For $\delta>0$ small enough and any $0<\epsilon<\epsilon_{0}$, there exists $\rho(\epsilon)>0$ such that for $0<\rho<\rho(\epsilon), G$ is well defined on $S_{\delta}$.

Proof. For fixed $\omega$ and $i$ and any $x^{c u}, x^{c u \prime} \in \mathcal{D}_{3}^{i} \times B(0, \epsilon)$, we have

$$
\begin{aligned}
& \left\|\xi^{c u}-\xi^{c u^{\prime}}\right\| \\
& =\left\|f_{i j}^{c u}\left(\theta^{-T} \omega, x^{c u}, u_{i}\left(\theta^{-T} \omega, x^{c u}\right)\right)-f_{i j}^{c u}\left(\theta^{-T} \omega, x^{c u^{\prime}}, u_{i}\left(\theta^{-T} \omega, x^{c u^{\prime}}\right)\right)\right\| \\
& \geq \frac{1}{Q}\left\|x^{c u}-x^{c u^{\prime}}\right\|-Q \delta\left\|x^{c u}-x^{c u^{\prime}}\right\| \\
& >\frac{1}{2 Q}\left\|x^{c u}-x^{c u^{\prime}}\right\|
\end{aligned}
$$

which implies that $f_{i j}^{c u}\left(\theta^{-T} \omega, \cdot, u_{i}\left(\theta^{-T} \omega, \cdot\right)\right)$ is one-to-one and thus is a homeomorphism from $\mathcal{D}_{3}^{i} \times B(0, \epsilon)$ to its range. Note that although the Mean Value Theorem does not hold in multi-dimensional space, we still have the first inequality above since the matrix of partial derivatives obtained by using the Mean Value Theorem componentwise is close to the Jacobian. Such estimates will be used repeatedly in our analysis.

Letting $x^{c}=x^{c \prime}$ and using Lemma 3.1, we have

$$
\begin{aligned}
& \left\|\xi^{u}-\xi^{u^{\prime}}\right\| \\
& =\left\|f_{i j}^{u}\left(\theta^{-T} \omega, x^{c}, u_{i}\left(\theta^{-T} \omega, x^{c}, x^{u}\right), x^{u}\right)-f_{i j}^{u}\left(\theta^{-T} \omega, x^{c}, u_{i}\left(\theta^{-T} \omega, x^{c}, x^{u \prime}\right), x^{u \prime}\right)\right\| \\
& \geq\left\|\left(D_{3} f_{i j}^{u}\right)^{-1}\right\|^{-1}\left\|x^{u}-x^{u^{\prime}}\right\|-\left\|D_{1} f_{i j}^{u}\right\|\left\|x^{c}-x^{c}\right\| \\
& \quad \quad-\left\|D_{2} f_{i j}^{u}\right\|\left\|u_{i}\left(x^{c}, x^{u}\right)-u_{i}\left(x^{c}, x^{u \prime}\right)\right\|+o\left(\left\|x^{c u}-x^{c u^{\prime}}\right\|\right) \\
& \geq 4\left\|x^{u}-x^{u \prime}\right\|-2 Q \delta\left\|x^{c u}-x^{c u^{\prime}}\right\| \\
& =(4-2 Q \delta)\left\|x^{u}-x^{u^{\prime}}\right\| \\
& >3\left\|x^{u}-x^{u \prime}\right\|,
\end{aligned}
$$

where by taking $\epsilon$ small enough, we have from $\left\|x^{u}-x^{u \prime}\right\|<2 \epsilon$ that

$$
o\left(\left\|x^{c u}-x^{c u^{\prime}}\right\|\right)<Q \delta\left\|x^{c u}-x^{c u^{\prime}}\right\| .
$$

For fixed $\delta, \epsilon>0$, there exists $\rho(\epsilon)>0$ such that for $0<\rho<\rho(\epsilon)$, we have

$$
\left\|f_{i j}^{u}\left(\theta^{-T} \omega, x^{c}, u_{i}\left(\theta^{-T} \omega, x^{c}, 0\right), 0\right)\right\|<\frac{1}{3} \epsilon,
$$

which together with (4.2) yield that

$$
\left\|f_{i j}^{u}\left(\theta^{-T} \omega, x^{c}, u_{i}\left(\theta^{-T} \omega, x^{c}, x^{u}\right), x^{u}\right)\right\|>\epsilon
$$

for $\frac{2}{3} \epsilon<\left\|x^{u}\right\|<\epsilon$. From this and the fact that $f_{i j}^{c u}\left(\theta^{-T} \omega, \cdot, u_{i}\left(\theta^{-T} \omega, \cdot\right)\right)$ is a homeomorphism, we conclude that $\bigcup_{k} \mathcal{D}_{3}^{k} \times B(0, \epsilon)$ is contained in the range of $f_{i j}^{c u}\left(\theta^{-T} \omega, \cdot, u_{i}\left(\theta^{T} \omega, \cdot\right)\right)$ for $i, j$ running all possible choices. This shows $G$ is well defined and completes the proof of the lemma.

Lemma 4.2. For all $0 \leq k \leq r$,

$$
\|B\|\left\|C^{-1}\right\|^{k}<\frac{1}{2}
$$


Proof. Using Lemma 3.1, we have

$$
\|B\|<\frac{1}{2}
$$

Writing

$$
C=\left(\begin{array}{cc}
D_{1} f_{i j}^{c}\left(\omega, x^{c}, x^{s}, x^{u}\right) & D_{3} f_{i j}^{c}\left(\omega, x^{c}, x^{s}, x^{u}\right) \\
D_{1} f_{i j}^{u}\left(\omega, x^{c}, x^{s}, x^{u}\right) & D_{3} f_{i j}^{u}\left(\omega, x^{c}, x^{s}, x^{u}\right)
\end{array}\right)=\left(\begin{array}{cc}
a & b \\
c & d
\end{array}\right),
$$

we have

$$
C^{-1}=\left(\begin{array}{ll}
\left(a-b d^{-1} c\right)^{-1} & -\left(a-b d^{-1} c\right)^{-1} b d^{-1} \\
\left(c a^{-1} b-d\right)^{-1} c a^{-1} & -\left(c a^{-1} b-d\right)^{-1}
\end{array}\right) .
$$

From Lemma 3.1, we know that

Hence,

$$
\begin{aligned}
& \|a\|<Q,\|c\|<\eta,\left\|a^{-1}\right\|<Q, \\
& \|b\|<\eta,\|d\|<Q,\left\|d^{-1}\right\|<\frac{1}{4} .
\end{aligned}
$$

$$
\begin{aligned}
\left\|\left(C^{-1}\right)_{11}\right\| & =\left\|\left(a-b d^{-1} c\right)^{-1}\right\| \\
& =\left\|\left(I-a^{-1} b d^{-1} c\right)^{-1} a^{-1}\right\| \\
& \leq\left\|a^{-1}\right\|\left(1+O\left(\eta^{2}\right)\right), \\
\left\|\left(C^{-1}\right)_{12}\right\| & =\left\|-\left(I-a^{-1} b d^{-1} c\right)^{-1} b d^{-1} a^{-1}\right\| \\
& \leq \frac{1}{4} Q \eta\left(1+O\left(\eta^{2}\right)\right)=O(\eta), \\
\left\|\left(C^{-1}\right)_{21}\right\| & =\left\|\left(d^{-1} c a^{-1} b-I\right)^{-1} d^{-1} c a^{-1}\right\| \\
& \leq \frac{1}{4} Q \eta\left(1+O\left(\eta^{2}\right)\right)=O(\eta), \\
\left\|\left(C^{-1}\right)_{22}\right\| & =\left\|-\left(d^{-1} c a^{-1} b-I\right)^{-1} d^{-1}\right\| \\
& \leq\left\|d^{-1}\right\|\left(1+O\left(\eta^{2}\right)\right) \leq \frac{1}{4}\left(1+O\left(\eta^{2}\right)\right),
\end{aligned}
$$

which yield that for $\eta$ sufficiently small,

$$
\left\|C^{-1}\right\|^{k}<2 \max \left(\left\|\left(D_{1} f_{i j}^{c}\right)^{-1}\right\|^{k}, \frac{1}{4}\right) .
$$

Therefore,

$$
\begin{aligned}
\|B\|\left\|C^{-1}\right\|^{k} & <2 \max \left(\left\|D_{2} f_{i j}^{s}\right\|\left\|\mid\left(D_{1} f_{i j}^{c}\right)^{-1}\right\|^{k}, \frac{1}{4}\left\|D_{2} f_{i j}^{s}\right\|\right) \\
& <2 \max \left(\frac{1}{4}, \frac{1}{4} \times \frac{1}{2}\right)=\frac{1}{2} .
\end{aligned}
$$

The proof of the lemma is complete.

We note that

$$
\|A\|<\eta
$$

and all $A, B, C, E$ and their inverses are bounded by $Q$, perhaps choosing a larger value of $Q$.

Remark. A, B, $C$ and $E$ depend on $i, j, x^{c u}, x^{s}, \omega, \psi(T)$ and $\phi(T, \omega)$. But all the above estimates hold uniformly. Note that in (4.4) the argument $i, j, \omega$ should be the same, while the argument $x^{c u}, x^{s}$ could vary as long as they vary only slightly. 
Lemma 4.3. For fixed $\delta$ small enough and any $0<\epsilon<\epsilon_{0}$, there exists a $\rho(\epsilon)>0$ such that for all $0<\rho<\rho(\epsilon), G$ maps $S_{\delta}$ to $S_{\delta}$.

Proof. It is sufficient to show that if $u \in S_{\delta}$, then

$$
\left\|(G u)_{j}\left(\omega, \xi^{c u}\right)-(G u)_{j}\left(\omega, \xi^{c u \prime}\right)\right\| \leq \delta\left\|\xi^{c u}-\xi^{c u \prime}\right\|
$$

for $\xi^{c u}$ and $\xi^{c u^{\prime}}$ in any sufficiently small neighborhood for all $\omega \in \Omega$ and $j$. This is because any two points $\xi^{c u}, \xi^{c u^{\prime}} \in \mathcal{D}_{3}^{j} \times B(0, \epsilon)$ are connected by a line segment which is covered by finitely many such neighborhoods and one can always choose a sequence of points $\xi_{0}=\xi^{c u}, \xi_{1}, \cdots, \xi_{m}=\xi^{c u^{\prime}}$ along the line segment such that for any $1 \leq k \leq m, \xi_{k}$ and $\xi_{k+1}$ are located in the same small neighborhood. Then

$\left\|(G u)_{j}\left(\omega, \xi^{c u}\right)-(G u)_{j}\left(\omega, \xi^{c u^{\prime}}\right)\right\| \leq \sum_{k} \delta\left\|\xi_{k}-\xi_{k-1}\right\|=\delta\left\|\xi_{m}-\xi_{0}\right\|=\delta\left\|\xi^{c u}-\xi^{c u^{\prime}}\right\|$.

Using Lemma 4.1, we have for $\xi^{c u^{\prime}}$ near $\xi^{c u}$ that there exists $x^{c u^{\prime}} \in \mathcal{D}_{3}^{i}$ near $x^{c u}$ such that

$$
\xi^{c u}=f_{i j}^{c u}\left(\theta^{-T} \omega, x^{c u}, u_{i}\left(\theta^{-T} \omega, x^{c u}\right)\right)
$$

and

$$
\xi^{c u \prime}=f_{i j}^{c u}\left(\theta^{-T} \omega, x^{c u^{\prime}}, u_{i}\left(\theta^{-T} \omega, x^{c u^{\prime}}\right)\right)
$$

Thus, we have

$$
\begin{aligned}
& \left\|(G u)_{j}\left(\omega, \xi^{c u}\right)-(G u)_{j}\left(\omega, \xi^{c u \prime}\right)\right\| \\
& =\left\|f_{i j}^{s}\left(\theta^{-T} \omega, x^{c u}, u_{i}\left(\theta^{-T} \omega, x^{c u}\right)\right)-f_{i j}^{s}\left(\theta^{-T} \omega, x^{c u \prime}, u_{i}\left(\theta^{-T} \omega, x^{c u \prime}\right)\right)\right\| \\
& \leq\|A\|\left\|x^{c u}-x^{c u^{\prime}}\right\|+\|B\| \delta\left\|x^{c u}-x^{c u^{\prime}}\right\|+o\left(\left\|x^{c u}-x^{c u^{\prime}}\right\|\right) \\
& \leq(2 \eta+\delta\|B\|)\left\|x^{c u}-x^{c u \prime}\right\| .
\end{aligned}
$$

Here, we have taken $\| x^{c u}-x^{c u \prime}||$ small enough so that $\left|o\left(\left\|x^{c u}-x^{c u \prime}\right\|\right)\right|<\eta \| x^{c u}-$ $x^{c u^{\prime}} \|$. Next we need to estimate $\left\|\xi^{c u}-\xi^{c u^{\prime}}\right\|$ in terms of $\left\|x^{c u}-x^{c u^{\prime}}\right\|$ :

$$
\begin{aligned}
\left\|\xi^{c u}-\xi^{c u \prime}\right\|= & \left\|f_{i j}^{c u}\left(\theta^{-T} \omega, x^{c u}, u_{i}\left(\theta^{-T} \omega, x^{c u}\right)\right)-f_{i j}^{c u}\left(\theta^{-T} \omega, x^{c u^{\prime}}, u_{i}\left(\theta^{-T} \omega, x^{c u^{\prime}}\right)\right)\right\| \\
\geq & \left\|f_{i j}^{c u}\left(\theta^{-T} \omega, x^{c u}, u_{i}\left(\theta^{-T} \omega, x^{c u}\right)\right)-f_{i j}^{c u}\left(\theta^{-T} \omega, x^{c u^{\prime}}, u_{i}\left(\theta^{-T} \omega, x^{c u}\right)\right)\right\| \\
& \quad-\left\|f_{i j}^{c u}\left(\theta^{-T} \omega, x^{c u^{\prime}}, u_{i}\left(\theta^{-T} \omega, x^{c u}\right)\right)-f_{i j}^{c u}\left(\theta^{-T} \omega, x^{c u^{\prime}}, u_{i}\left(\theta^{-T} \omega, x^{c u \prime}\right)\right)\right\| \\
\geq & \left\|C^{-1}\right\|^{-1}\left\|x^{c u}-x^{c u \prime}\right\|+o\left(\left\|x^{c u}-x^{c u \prime}\right\|\right)-\|E\| \delta\left\|x^{c u}-x^{c u^{\prime}}\right\| \\
\geq & \left\|C^{-1}\right\|^{-1}\left(1-2 \delta Q^{2}\right)\left\|x^{c u}-x^{c u^{\prime}}\right\| .
\end{aligned}
$$

Here again we have taken $\left\|x^{c u}-x^{c u^{\prime}}\right\|$ small enough so that $\left|o\left(\| x^{c u}-x^{c u \prime}||\right)\right|<$ $\delta Q\left\|x^{c u}-x^{c u^{\prime}}\right\|$. Then, we have

$$
\begin{gathered}
\left\|(G u)_{j}\left(\omega, \xi^{c u}\right)-(G u)_{j}\left(\omega, \xi^{c u^{\prime}}\right)\right\| \leq(2 \eta+\delta\|B\|)\left\|x^{c u}-x^{c u \prime}\right\| \\
\leq(2 \eta+\delta\|B\|) \frac{\left\|C^{-1}\right\|}{\left(1-2 \delta Q^{2}\right)}\left\|\xi^{c u}-\xi^{c u^{\prime}}\right\| \\
\leq \frac{2 \eta Q+\frac{1}{2} \delta}{1-2 \delta Q^{2}}\left\|\xi^{c u}-\xi^{c u \prime}\right\| \leq \frac{3}{4} \delta\left\|\xi^{c u}-\xi^{c u \prime}\right\|,
\end{gathered}
$$

provided $\delta<\frac{1}{12 Q^{2}}$ and $\eta<\frac{\delta}{16 Q}$. So $G$ maps $S_{\delta}$ to $S_{\delta}$. This completes the proof of the lemma.

Lemma 4.4. If $\delta, \eta>0$ are small enough, there exists $\epsilon(\eta)>0$ such that for any $0<\epsilon<\epsilon(\eta)$ there exists $\rho(\epsilon)>0$ such that for $0<\rho<\rho(\epsilon), G$ is a contraction on $S_{\delta}$. 
Proof. Let $u, u^{\prime} \in S_{\delta}$. Fix $\omega \in \Omega$ and let $\xi^{c u} \in\left(\sigma_{j} \times \tau_{j}^{u}\right)\left(\tilde{E}_{\epsilon}^{u} \mid U_{3}^{j}\right)$ be given. By Lemma 4.1, there exist $i$ and $x^{c u}, x^{c u^{\prime}} \in \mathcal{D}_{3}^{i} \times B(0, \epsilon)$ such that

$$
\xi^{c u}=f_{i j}^{c}\left(\theta^{-T} \omega, x^{c u}, u_{i}\left(\theta^{-T} \omega, x^{c u}\right)\right)=f_{i j}^{c}\left(\theta^{-T} \omega, x^{c u \prime}, u_{i}^{\prime}\left(\theta^{-T} \omega, x^{c u \prime}\right)\right) .
$$

Here, as long as $\epsilon$ is small, $x^{c u}, x^{c u \prime}$ are close enough to be in the same $\mathcal{D}_{3}^{i} \times B(0, \epsilon)$. Then if $\epsilon$ is small enough, we have that $o\left(\left\|x^{c u}-x^{c u^{\prime}}\right\|\right)$ and $o\left(\left\|u-u^{\prime}\right\|\right)$ are so small that

$$
\begin{gathered}
\left\|(G u)_{j}\left(\omega, \xi^{c u}\right)-\left(G u^{\prime}\right)_{j}\left(\omega, \xi^{c u}\right)\right\| \\
=\left\|f_{i j}^{s}\left(\theta^{-T} \omega, x^{c u}, u_{i}\left(\theta^{-T} \omega, x^{c u}\right)\right)-f_{i j}^{s}\left(\theta^{-T} \omega, x^{c u^{\prime}}, u_{i}^{\prime}\left(\theta^{-T} \omega, x^{c u^{\prime}}\right)\right)\right\| \\
\leq\|A\|\left\|x^{c u}-x^{c u^{\prime}}\right\|+\|B\|\left\|u_{i}\left(\theta^{-T} \omega, x^{c u}\right)-u_{i}^{\prime}\left(\theta^{-T} \omega, x^{c u}\right)\right\| \\
+Q\left\|u_{i}^{\prime}\left(\theta^{-T} \omega, x^{c u}\right)-u_{i}^{\prime}\left(\theta^{-T} \omega, x^{c u^{\prime}}\right)\right\|+o\left(\left\|x^{c u}-x^{c u^{\prime}}\right\|\right)+o\left(\left\|u-u^{\prime}\right\|\right) \\
\leq(\eta+2 \delta Q)\left\|x^{c u}-x^{c u^{\prime}}\right\|+\frac{2}{3}\left\|u_{i}\left(\theta^{-T} \omega\right)-u_{i}^{\prime}\left(\theta^{-T} \omega\right)\right\| .
\end{gathered}
$$

Next, we need to estimate $\left\|x^{c u}-x^{c u^{\prime}}\right\|$ in terms of $\left\|u_{i}\left(\theta^{-T} \omega\right)-u_{i}^{\prime}\left(\theta^{-T} \omega\right)\right\|$. We first have

$$
\begin{aligned}
& \left\|f_{i j}^{c u}\left(\theta^{-T} \omega, x^{c u}, u_{i}\left(\theta^{-T} \omega, x^{c u}\right)\right)-f_{i j}^{c u}\left(\theta^{-T} \omega, x^{c u \prime}, u_{i}\left(\theta^{-T} \omega, x^{c u}\right)\right)\right\| \\
& \geq Q^{-1}\left\|x^{c u}-x^{c u \prime}\right\|
\end{aligned}
$$

and

$$
\begin{aligned}
& \left\|f_{i j}^{c u}\left(\theta^{-T} \omega, x^{c u^{\prime}}, u_{i}^{\prime}\left(\theta^{-T} \omega, x^{c u^{\prime}}\right)\right)-f_{i j}^{c u}\left(\theta^{-T} \omega, x^{c u^{\prime}}, u_{i}\left(\theta^{-T} \omega, x^{c u}\right)\right)\right\| \\
& \leq Q\left(\delta\left\|x^{c u}-x^{c u^{\prime}}\right\|+\left\|u_{i}\left(\theta^{-T} \omega\right)-u_{i}^{\prime}\left(\theta^{-T} \omega\right)\right\|\right) .
\end{aligned}
$$

Noting that

$$
\xi^{c u}=f_{i j}^{c u}\left(\theta^{-T} \omega, x^{c u}, u_{i}\left(\theta^{-T} \omega, x^{c u}\right)\right)=f_{i j}^{c u}\left(\theta^{-T} \omega, x^{c u^{\prime}}, u_{i}^{\prime}\left(\theta^{-T} \omega, x^{c u \prime}\right)\right),
$$

we combine the above estimates to obtain

$$
\left\|x^{c u}-x^{c u^{\prime}}\right\| \leq\left(2 Q^{2}(\eta+2 \delta Q)+\frac{2}{3}\right)\left\|u_{i}\left(\theta^{-T} \omega\right)-u_{i}^{\prime}\left(\theta^{-T} \omega\right)\right\| .
$$

Taking $\delta, \eta$ small enough, then we get

$$
\left\|(G u)_{j}\left(\omega, \xi^{c u}\right)-\left(G u^{\prime}\right)_{j}\left(\omega, \xi^{c u}\right)\right\| \leq \frac{3}{4}\left\|u_{i}\left(\theta^{-T} \omega\right)-u_{i}^{\prime}\left(\theta^{-T} \omega\right)\right\| .
$$

Proof of Proposition 4.1. Using Lemma 4.4, by the uniform contraction principle, $G$ has a unique fixed point in $S_{\delta}$, call it $u$. If $u^{\prime} \in S$ is such that

$$
\phi(T, \omega)\left(\operatorname{graph} u^{\prime}(\omega)\right) \supset \operatorname{graph} u^{\prime}\left(\theta^{T} \omega\right),
$$

then the proof of Lemma 4.4 can be applied: Fix $\omega \in \Omega$ and let $\xi^{c u} \in\left(\sigma_{j} \times\right.$ $\left.\tau_{j}^{u}\right)\left(\tilde{E}_{\epsilon}^{u} \mid U_{3}^{j}\right)$ be given. Since $u^{\prime}$ is invariant, there exist $i$ and $x^{c u^{\prime}} \in \mathcal{D}_{3}^{i} \times B(0, \epsilon)$ such that

$$
\xi^{c u}=f_{i j}^{c u}\left(\theta^{-T} \omega, x^{c u}, u_{i}\left(\theta^{-T} \omega, x^{c u}\right)\right)=f_{i j}^{c u}\left(\theta^{-T} \omega, x^{c u^{\prime}}, u_{i}^{\prime}\left(\theta^{-T} \omega, x^{c u \prime}\right)\right) .
$$

Here, as long as $\epsilon$ is small, $x^{c u}, x^{c u \prime}$ are close enough to be in the same $\mathcal{D}_{3}^{i} \times$ $B(0, \epsilon)$. Then

$$
\begin{gathered}
\left\|u_{j}\left(\omega, \xi^{c u}\right)-u_{j}^{\prime}\left(\omega, \xi^{c u}\right)\right\| \\
=\left\|f_{i j}^{s}\left(\theta^{-T} \omega, x^{c u}, u_{i}\left(\theta^{-T} \omega, x^{c u}\right)\right)-f_{i j}^{s}\left(\theta^{-T} \omega, x^{c u^{\prime}}, u_{i}^{\prime}\left(\theta^{-T} \omega, x^{c u^{\prime}}\right)\right)\right\| \\
\leq(\eta+2 \delta Q)\left\|x^{c u}-x^{c u^{\prime}}\right\|+\frac{2}{3}\left\|u_{i}\left(\theta^{-T} \omega\right)-u_{i}^{\prime}\left(\theta^{-T} \omega\right)\right\| .
\end{gathered}
$$


Similarly, as in Lemma 4.4 we get

$$
\left\|x^{c u}-x^{c u^{\prime}}\right\| \leq\left(2 Q^{2}(\eta+2 \delta Q)+\frac{2}{3}\right)\left\|u_{i}\left(\theta^{-T} \omega\right)-u_{i}^{\prime}\left(\theta^{-T} \omega\right)\right\| .
$$

So

$$
\left\|u_{j}\left(\omega, \xi^{c u}\right)-u_{j}^{\prime}\left(\omega, \xi^{c u}\right)\right\| \leq \frac{3}{4}\left\|u_{i}\left(\theta^{-T} \omega\right)-u_{i}^{\prime}\left(\theta^{-T} \omega\right)\right\| .
$$

This shows that $u=u^{\prime}$, giving the uniqueness in $S$.

To show

$$
\phi(t, \omega)(\operatorname{graph} u(\omega)) \supset \operatorname{graph} u\left(\theta^{t} \omega\right),
$$

we use the uniqueness of $u$.

For any fixed $t>T$, we can define $G^{\prime}$ on $S_{\delta}$ based on $t$ just as $G$ is based on $T$. $G^{\prime}$ is well defined. Then we have that $\phi(t, \omega)(\operatorname{graph} u(\omega)) \cap \tilde{E}_{\epsilon}$ is the graph of an element $\bar{u} \in S_{\delta}$ at the $\theta^{t} \omega$ fiber.

Since for each $\omega$

$$
\phi\left(T, \theta^{-T} \omega\right)\left(\operatorname{graph} u\left(\theta^{-T} \omega\right)\right) \supset \operatorname{graph} u(\omega),
$$

we have

$$
\begin{aligned}
\phi(t, \omega)(\operatorname{graph} u(\omega)) & \subset \phi(t, \omega) \phi\left(T, \theta^{-T} \omega\right)\left(\operatorname{graph} u\left(\theta^{-T} \omega\right)\right) \\
& =\phi\left(T, \theta^{t-T} \omega\right) \phi\left(t, \theta^{-T} \omega\right)\left(\operatorname{graph} u\left(\theta^{-T} \omega\right)\right)
\end{aligned}
$$

which gives

$$
\operatorname{graph} \bar{u}\left(\theta^{t} \omega\right) \subset \phi\left(T, \theta^{-T}\left(\theta^{t} \omega\right)\right)\left(\operatorname{graph} \bar{u}\left(\theta^{-T}\left(\theta^{t} \omega\right)\right)\right),
$$

or equivalently,

$$
\operatorname{graph} \bar{u}(\tilde{\omega}) \subset \phi\left(T, \theta^{-T}(\tilde{\omega})\right)\left(\operatorname{graph} \bar{u}\left(\theta^{-T}(\tilde{\omega})\right)\right) .
$$

Comparing (4.8) and (4.9) and using the uniqueness of the fixed point of $G$, we obtain

$$
\bar{u}=u \text {. }
$$

This completes the proof of the proposition.

\section{Smoothness of the RAndom unstable manifolds}

In this section, we prove the smoothness of the random unstable manifold. Let $u \in S_{\delta}$ denote the unique fixed point of $G$. So the graph of $u(\omega)$ is the unique random unstable manifold $\tilde{\mathcal{W}}^{u}(\omega)$. We prove $u$ is $C^{r}$. We construct linear functions in the local coordinates as the candidates for $D u_{i}(\omega)$ and prove they are indeed the derivatives of $u_{i}(\omega)$.

In the local coordinates, for any fixed $\omega, u(\omega)$ is represented by $u_{i}(\omega):\left(\mathcal{D}_{3}^{i} \times\right.$ $B(0, \epsilon)) \rightarrow \mathbb{R}^{k}$, for $i=1, \cdots, s$. If $u(\omega) \in C^{1}, D u_{i}(\omega)$ assigns to each point in $\left(\mathcal{D}_{3}^{i} \times B(0, \epsilon)\right)$ a linear map from $\mathbb{R}^{n-k}$ to $\mathbb{R}^{k}$. Thus $D u(\omega)$ is represented by

$$
v_{i}(\omega) \in C^{0}\left(\left(\mathcal{D}_{3}^{i} \times B(0, \epsilon)\right), L\left(\mathbb{R}^{n-k}, \mathbb{R}^{k}\right)\right),
$$

for $i=1, \cdots, s$. The candidates for $D u(\omega)$ are of the form

$$
v(\omega)=\left(v_{1}(\omega), \cdots, v_{s}(\omega)\right) \in \prod_{i=1}^{s} C^{0}\left(\left(\mathcal{D}_{3}^{i} \times B(0, \epsilon)\right), L\left(\mathbb{R}^{n-k}, \mathbb{R}^{k}\right)\right),
$$

and we denote the space of such mappings by $\overline{T S}$. If $v(\omega)$ is such an $s$-tuple, define

$$
\|v(\omega)\|=\max _{1 \leq i \leq s} \sup _{x^{c u} \in\left(\mathcal{D}_{3}^{i} \times B(0, \epsilon)\right)}\left\|v_{i}\left(\omega, x^{c u}\right)\right\|,
$$


if this exists, where $\|\cdot\|$ is the operator norm. For $v=\{v(\omega) \in \overline{T S}: \omega \in \Omega\}$, define

$$
\|v\|:=\sup _{\omega}\|v(\omega)\| .
$$

Let $T S$ be the space of all such random linear mappings $v$. To be more clear, any element $v$ of $T S$ can first be viewed as a function of $\omega \in \Omega$, and for any fixed $\omega, v(\omega)$ is an s-tuple $\left(v_{1}(\omega), \cdots, v_{s}(\omega)\right) \in \prod_{i=1}^{s} C^{0}\left(\left(\mathcal{D}_{3}^{i} \times B(0, \epsilon)\right), L\left(\mathbb{R}^{n-k}, \mathbb{R}^{k}\right)\right)$. Define the norm $\|\cdot\|$ on $T S$ by (5.1) and (5.2). Under this norm, $T S$ is complete.

In terms of the local coordinates, $u(\omega)$ satisfies

$$
u_{j}\left(\theta^{T} \omega, \xi^{c u}\right)=f_{i j}^{s}\left(\omega, x^{c u}, u_{i}\left(\omega, x^{c u}\right)\right),
$$

where $\xi^{c u}=f_{i j}^{c u}\left(\omega, x^{c u}, u_{i}\left(\omega, x^{c u}\right)\right)$. Differentiating these formally, we have

$$
v_{j}\left(\theta^{T} \omega, \xi^{c u}\right)\left[C+E v_{i}\left(\omega, x^{c u}\right)\right]=A+B v_{i}\left(\omega, x^{c u}\right),
$$

where $A=D_{1,3} f_{i j}^{s}, B=D_{2} f_{i j}^{s}, C=D_{1,3} f_{i j}^{c u}$ and $E=D_{2} f_{i j}^{c u}$, the arguments of $A, B, C, E$, are all $\left(\omega, x^{c u}, u_{i}\left(\omega, x^{c u}\right)\right)$. So

$$
v_{j}\left(\theta^{T} \omega, \xi^{c u}\right)=H_{i j}(\omega) v_{i}\left(\omega, x^{c u}\right),
$$

where

$$
H_{i j}(\omega) v_{i}\left(\omega, x^{c u}\right)=\left[A+B v_{i}\left(\omega, x^{c u}\right)\right]\left[C+E v_{i}\left(\omega, x^{c u}\right)\right]^{-1} .
$$

Thus, (5.3) induces an operator $H$ on TS.

Remark. In the definition of $H_{i j}$, the argument of all $A, B, C, E$ are all $\left(\omega, x^{c u}\right.$, $u_{i}\left(\omega, x^{c u}\right)$ ), while later in the computation of contraction, the argument of $A, B, C, E$ may be different, and in fact the matrices themselves may be different, being small perturbations of these Jacobians. However, this does not affect the estimates.

We want to prove that $H$ has a fixed point $v(\omega)$. For technical reasons, we need a smooth partition of unity. Choose $C^{r}$ functions: $h_{i}: \hat{U}_{3}^{i} \rightarrow[0,1]$ with support of $h_{i} \subset \hat{U}_{2}^{i}$ and $\sum_{i=1}^{s} h_{i}=1$ on $\bigcup_{i}^{s} \hat{U}_{1}^{i}$. Here, $\hat{U}_{k}^{i}:=\left(\sigma_{i} \times \tau_{i}^{u}\right)^{-1}\left(\mathcal{D}_{k}^{i} \times B(0, \epsilon)\right)$. Define

$$
v_{i}^{0}\left(\omega, x^{c u}\right)=0, i=1,2, \cdots, s,
$$

and

where

$$
v_{j}^{n+1}\left(\theta^{T} \omega, \xi^{c u}\right)=\sum_{i=1}^{s} h_{i}\left(m_{-}\right) H_{i j}(\omega) v_{i}^{n}\left(\omega, x^{c u}\right)
$$

$$
\phi\left(T, \omega, u\left(\omega, m_{-}\right)\right)=u\left(\theta^{T} \omega, \sigma_{j}^{-1}\left(\xi^{c u}\right)\right) .
$$

Then $m_{-} \in \bigcup_{i}^{s} \hat{U}_{1}^{i}$ and $\sum_{i=1}^{s} h_{i}\left(m_{-}\right)=1$. We will show $\left\{v^{n}\right\}$ converges to a fixed point of (5.3).

Proposition 5.1. The sequence $\left\{v^{n}(\omega)\right\}$ converges to a solution of the equations

$$
v_{j}\left(\theta^{T} \omega\right)=\sum_{i=1}^{s} h_{i} \cdot H_{i j}(\omega) v_{i}(\omega)
$$

First, we claim

Lemma 5.1. $\left\|v_{i}^{n}\left(\omega, x^{c u}\right)\right\|<\delta$ for all $n$ and $\omega$. 
Proof. It is sufficient to show

$$
\left\|H_{i j}(\omega) v_{i}^{n}(\omega)\right\|<\delta
$$

Letting $\delta>0$ be such that $\delta Q^{2} \ll 1$, for $\left\|v_{i}^{n}(\omega)\right\|<\delta$ we have

$$
\begin{aligned}
\left\|\left[C+E v_{i}^{n}(\omega)\right]^{-1}\right\| & =\left\|\left[C\left(1+C^{-1} E v_{i}^{n}\right)\right]^{-1}\right\| \\
& =\left\|\left[1-\left(-C^{-1} E v_{i}^{n}\right)\right]^{-1} C^{-1}\right\|=\left\|\sum_{k=0}^{\infty}\left(-C^{-1} E v_{i}^{n}\right)^{k} C^{-1}\right\| \\
& \leq \sum_{k=0}^{\infty}\left\|C^{-1} E v_{i}^{n}\right\|^{k}\left\|C^{-1}\right\| \leq \sum_{k=0}^{\infty}\left\|\delta Q^{2}\right\|^{k}\left\|C^{-1}\right\|=\frac{\left\|C^{-1}\right\|}{1-\delta Q^{2}} .
\end{aligned}
$$

Thus, using (5.3), by induction we have

$$
\left\|H_{i j}(\omega) v_{i}^{n}(\omega)\right\| \leq(\eta+\|B\| \delta) \frac{\left\|C^{-1}\right\|}{1-\delta Q^{2}} \leq \frac{Q \eta+\frac{1}{2} \delta}{1-\delta Q^{2}} \leq \delta,
$$

provided $\eta$ is small enough. This completes the proof of the lemma.

Lemma 5.2. $\left\|v^{n+1}\left(\theta^{T} \omega\right)-v^{n}\left(\theta^{T} \omega\right)\right\| \leq \lambda\left\|v^{n}(\omega)-v^{n-1}(\omega)\right\|$ for some $0<\lambda<1$.

Proof. It is sufficient to show

$$
\left\|H_{i j}(\omega) v_{i}^{n}(\omega)-H_{i j}(\omega) v_{i}^{n-1}(\omega)\right\| \leq \lambda\left\|v^{n}(\omega)-v^{n-1}(\omega)\right\| \text { for all } i, j .
$$

First, we note that

$$
\begin{aligned}
& H_{i j}(\omega) v_{i}^{n}(\omega)-H_{i j}(\omega) v_{i}^{n-1}(\omega) \\
= & {\left[A+B v_{i}^{n}(\omega)\right]\left[C+E v_{i}^{n}(\omega)\right]^{-1} } \\
& -\left[A+B v_{i}^{n-1}(\omega)\right]\left[C+E v_{i}^{n-1}(\omega)\right]^{-1} \\
= & \left(A+B v_{i}^{n}\right)\left(C+E v_{i}^{n}\right)^{-1}\left[\left(C+E v_{i}^{n-1}\right)-\left(C+E v_{i}^{n}\right)\right]\left(C+E v_{i}^{n-1}\right)^{-1} \\
& +\left[\left(A+B v_{i}^{n}\right)-\left(A+B v_{i}^{n-1}\right)\right]\left(C+E v_{i}^{n-1}\right)^{-1} .
\end{aligned}
$$

Estimating the above and using Lemma 4.2, we have

$$
\begin{aligned}
& \left\|H_{i j}(\omega) v_{i}^{n}(\omega)-H_{i j}(\omega) v_{i}^{n-1}(\omega)\right\| \\
\leq \quad & (\eta+\|B\| \delta) \frac{\left\|C^{-1}\right\|}{1-\delta Q^{2}} Q\left\|v_{i}^{n}-v_{i}^{n-1}\right\| \frac{\left\|C^{-1}\right\|}{1-\delta Q^{2}} \\
& +\|B\|\left\|v_{i}^{n}-v_{i}^{n-1}\right\| \frac{\left\|C^{-1}\right\|}{1-\delta Q^{2}} \\
= & \left((\eta+\|B\| \delta) \frac{\left\|C^{-1}\right\|^{2}}{\left(1-\delta Q^{2}\right)^{2}} Q+\frac{\frac{1}{2}}{1-\delta Q^{2}}\right)\left\|v^{n}(\omega)-v^{n-1}(\omega)\right\| \\
= & \lambda\left\|v^{n}(\omega)-v^{n-1}(\omega)\right\|,
\end{aligned}
$$

for all $i, j$. By choosing $\eta$ and $\delta$ small enough, we have $\lambda<1$. The proof is complete.

By the contraction principle, $\left\{v^{n}\right\}$ converges to $v$, which satisfies (5.4).

We are ready to prove that $v$ is the derivative of $u$.

Proposition 5.2. For each $\xi^{c u} \in\left(\mathcal{D}_{3}^{j} \times B(0, \epsilon)\right), D u_{j}\left(\theta^{T} \omega, \xi^{c u}\right)$ exists and equals $v_{j}\left(\theta^{T} \omega, \xi^{c u}\right)$. Hence, $u \in C^{1}$ and $v_{j}\left(\theta^{T} \omega, \xi^{c u}\right)=H_{i j}(\omega) v_{i}\left(\omega, x^{c u}\right)$. 
Proof. For a fixed $\omega \in \Omega$, we define an increasing function $\gamma_{\omega}:(0,1) \rightarrow \mathbb{R}$ :

$$
\begin{aligned}
\gamma_{\theta^{T} \omega}(a)= & \max _{i} \sup _{\xi^{c u}, \xi^{c u^{\prime}} \in\left(\mathcal{D}_{3}^{i} \times B(0, \epsilon)\right), 0<\left\|\xi^{c u}-\xi^{c u} \prime\right\|<a} \\
& \times \frac{\left\|u_{i}\left(\theta^{T} \omega, \xi^{c u^{\prime}}\right)-u_{i}\left(\theta^{T} \omega, \xi^{c u}\right)-v_{i}\left(\theta^{T} \omega, \xi^{c u}\right)\left(\xi^{c u^{\prime}}-\xi^{c u}\right)\right\|}{\left\|\xi^{c u^{\prime}}-\xi^{c u}\right\|} .
\end{aligned}
$$

Note that $\gamma_{\theta^{T} \omega}$ is bounded by $2 \delta$.

We want to show $\gamma_{\omega}(a) \rightarrow 0$ as $a \rightarrow 0$. To prove this, we claim

Claim. $\gamma_{\omega}(a)$ satisfies

$$
\gamma_{\theta^{T} \omega}(a) \leq s \gamma_{\omega}(z a)+r(\omega, a)
$$

for small $a$, for some $0 \leq s<1, z>1$, where $r(\omega, a)$ is a decreasing function which approaches zero as $a \rightarrow 0$ uniformly in $\omega \in \Omega$.

Proof of the Claim. Let $\xi^{c u} \in\left(\mathcal{D}_{3}^{j} \times B(0, \epsilon)\right)$. By Lemma 4.1. we have $\xi^{c u}=$ $f_{i j}^{c u}\left(\omega, x^{c u}, u_{i}\left(\omega, x^{c u}\right)\right)$ for some $x^{c u} \in\left(\mathcal{D}_{2}^{i} \times B(0, \epsilon)\right)$. We choose $d \in(0, a)$ so small that if $\xi^{c u^{\prime}} \in\left(\mathcal{D}_{3}^{j} \times B(0, \epsilon)\right)$ with $\left\|\xi^{c u}-\xi^{c u^{\prime}}\right\|<d$, then there exists $x^{c u \prime} \in$ $\left(\mathcal{D}_{3}^{i} \times B(0, \epsilon)\right)$ such that $\xi^{c u^{\prime}}=f_{i j}^{c}\left(\omega, x^{c u^{\prime}}, u_{i}\left(\omega, x^{c u^{\prime}}\right)\right)$.

To show

$$
\gamma_{\theta^{T} \omega}(a) \leq s \gamma_{\omega}(z a)+r(\omega, a)
$$

it is sufficient to show that

$$
\begin{aligned}
& \left\|u_{j}\left(\theta^{T} \omega, \xi^{c u^{\prime}}\right)-u_{j}\left(\theta^{T} \omega, \xi^{c u}\right)-H_{i j}(\omega) v_{i}\left(\theta^{T} \omega, \xi^{c u}\right) \cdot\left(\xi^{c u \prime}-\xi^{c u}\right)\right\| \\
& \quad \leq\left[s \gamma_{\omega}(z a)+r(a)\right]\left\|\xi^{c u \prime}-\xi^{c u}\right\|
\end{aligned}
$$

for all $\xi^{c u}, \xi^{c u \prime}, i, j$ as above and $\left\|\xi^{c u^{\prime}}-\xi^{c u}\right\| \leq a<d$.

First, we have

$$
\begin{aligned}
& \xi^{c u^{\prime}}-\xi^{c u}=f_{i j}^{c u}\left(\omega, x^{c u^{\prime}}, u_{i}\left(\omega, x^{c u^{\prime}}\right)\right)-f_{i j}^{c u}\left(\omega, x^{c u}, u_{i}\left(\omega, x^{c u}\right)\right) \\
&=C\left(x^{c u^{\prime}}-x^{c u}\right)+E\left(u_{i}\left(\omega, x^{c u^{\prime}}\right)-u_{i}\left(\omega, x^{c u}\right)\right)+o\left(\left\|x^{c u^{\prime}}-x^{c u}\right\|\right), \\
& u_{j}\left(\theta^{T} \omega, \xi^{c u^{\prime}}\right)-u_{j}\left(\theta^{T} \omega, \xi^{c u}\right)= f_{i j}^{s}\left(\omega, x^{c u^{\prime}}, u_{i}\left(\omega, x^{c u^{\prime}}\right)\right)-f_{i j}^{s}\left(\omega, x^{c u}, u_{i}\left(\omega, x^{c u}\right)\right) \\
&= A\left(x^{c u^{\prime}}-x^{c u}\right)+B\left(u_{i}\left(\omega, x^{c u \prime}\right)-u_{i}\left(\omega, x^{c u}\right)\right) \\
&+o\left(\left\|x^{c u^{\prime}}-x^{c u}\right\|\right) .
\end{aligned}
$$

Note that the quantity $o\left(\left\|x^{c u}-x^{c u^{\prime}}\right\|\right)$ is uniformly in $\omega \in \Omega$. From the first equation it follows that

$$
\left\|x^{c u^{\prime}}-x^{c u}\right\| \leq\left\|C^{-1}\right\| \frac{\left\|\xi^{c u^{\prime}}-\xi^{c u}\right\|}{1-2 \delta Q^{2}}
$$

and that

$$
\begin{aligned}
\xi^{c u^{\prime}}-\xi^{c u}= & {\left[C+E v_{i}\left(\omega, x^{c u}\right)\right]\left(x^{c u^{\prime}}-x^{c u}\right) } \\
& +E\left[u_{i}\left(\omega, x^{c u^{\prime}}\right)-u_{i}\left(\omega, x^{c u}\right)-v_{i}\left(\omega, x^{c u}\right)\left(x^{c u^{\prime}}-x^{c u}\right)\right]+o\left(\left\|x^{c u^{\prime}}-x^{c u}\right\|\right) .
\end{aligned}
$$


Hence,

$$
\begin{aligned}
&\left\|u_{j}\left(\theta^{T} \omega, \xi^{c u^{\prime}}\right)-u_{j}\left(\theta^{T} \omega, \xi^{c u}\right)-H_{i j}(\omega) v_{i}\left(\theta^{T} \omega, \xi^{c u}\right)\left(\xi^{c u^{\prime}}-\xi^{c u}\right)\right\| \\
&= \| A\left(x^{c u^{\prime}}-x^{c u}\right)+B\left(u_{i}\left(\omega, x^{c u^{\prime}}\right)-u_{i}\left(\omega, x^{c u}\right)\right)+o\left(\left\|x^{c u^{\prime}}-x^{c u}\right\|\right) \\
&-\left[A+B v_{i}\left(\omega, x^{c u}\right)\right]\left[C+E v_{i}\left(\omega, x^{c u}\right)\right]^{-1} \cdot\left(\xi^{c u^{\prime}}-\xi^{c u}\right) \| \\
&= \| A\left(x^{c u^{\prime}}-x^{c u}\right)+B\left[u_{i}\left(\omega, x^{c u^{\prime}}\right)-u_{i}\left(\omega, x^{c u}\right)\right]-\left[A+B v_{i}\left(\omega, x^{c u}\right)\right]\left(x^{c u^{\prime}}-x^{c u}\right) \\
&-\left[A+B v_{i}\left(\omega, x^{c u}\right)\right]\left[C+E v_{i}\left(\omega, x^{c u}\right)\right]^{-1} E\left[u_{i}\left(\omega, x^{c u^{\prime}}\right)-u_{i}\left(\omega, x^{c u}\right)\right. \\
&\left.-v_{i}\left(\omega, x^{c u}\right)\left(x^{c u^{\prime}}-x^{c u}\right)\right]+o\left(\left\|x^{c u^{\prime}}-x^{c u}\right\|\right) \| \\
&= \|\left\{B-\left[A+B v_{i}\left(\omega, x^{c u}\right)\right]\left[C+E v_{i}\left(\omega, x^{c u}\right)\right]^{-1} \cdot E\right\}\left[u_{i}\left(\omega, x^{c u^{\prime}}\right)-u_{i}\left(\omega, x^{c u}\right)\right. \\
&\left.-v_{i}\left(\omega, x^{c u}\right)\left(x^{c u^{\prime}}-x^{c u}\right)\right]+o\left(\left\|x^{c u^{\prime}}-x^{c u}\right\|\right) \| \\
& \leq {[\|B\|+O(\eta+\delta)] \gamma_{\omega}\left(\left\|x^{c u \prime}-x^{c u}\right\|\right)\left\|x^{c u^{\prime}}-x^{c u}\right\|+\left\|o\left(\left\|x^{c u^{\prime}}-x^{c u}\right\|\right)\right\| } \\
& \leq \frac{[\|B\|+O(\eta+\delta)]\left\|C^{-1}\right\|}{1-2 \delta Q^{2}} \gamma_{\omega}\left(\left\|x^{c u \prime}-x^{c u}\right\|\right)\left\|\xi^{c u \prime}-\xi^{c u}\right\|+\left\|o\left(\left\|x^{c u^{\prime}}-x^{c u}\right\|\right)\right\| .
\end{aligned}
$$

Taking $s=\frac{\|B\|+O(\eta+\delta)\left\|C^{-1}\right\|}{1-2 \delta Q^{2}}$, if $\eta$ and $\delta$ are small enough, then $s<1$. Let $z=\frac{Q}{1-2 \delta Q^{2}}$. Then

$$
\left\|x^{c u^{\prime}}-x^{c u}\right\| \leq z\left\|\xi^{c u^{\prime}}-\xi^{c u}\right\|<z d<z a
$$

and then

$$
\gamma_{\omega}\left(\left\|x^{c u^{\prime}}-x^{c u}\right\|\right) \leq \gamma_{\omega}(z a)
$$

Since

$$
\left\|\xi^{c u \prime}-\xi^{c u}\right\| \geq z^{-1}\left\|x^{c u \prime}-x^{c u}\right\|,
$$

$o\left(\left\|x^{c u^{\prime}}-x^{c u}\right\|\right)$ can be bounded by $r(\omega, a)\left\|\xi^{c u^{\prime}}-\xi^{c u}\right\|$, where $r(\omega, a) \rightarrow 0$ uniformly in $\omega \in \Omega$ as $a \rightarrow 0$. This shows

$$
\gamma_{\theta^{T} \omega}(a) \leq s \gamma_{\omega}(z a)+r(\omega, a) .
$$

Note that $z$ can be taken to be larger than 1 because $\gamma_{\omega}(a)$ is increasing in $a$. This completes the proof of the Claim.

We are ready to show $\gamma_{\omega}(a) \rightarrow 0$ as $a \rightarrow 0$.

Replace $a$ successively by $a z^{-1}, a z^{-2}, \cdots, a z^{-n}$, replace $\omega$ successively by $\omega, \theta^{T} \omega$, $\cdots, \theta^{(n-1) T} \omega$, weigh the terms with $s^{n-1}, s^{n-2}, \cdots, 1$ and add them together to get

$$
\begin{aligned}
\gamma_{\theta^{n T} \omega}\left(a z^{-n}\right) \leq & s^{n} \gamma_{\omega}(a)+r\left(\theta^{(n-1) T \omega}, a z^{-n}\right)+\operatorname{sr}\left(\theta^{(n-2) T \omega}, a z^{-n+1}\right) \\
& +\cdots+s^{n-1} r\left(\omega, a z^{-1}\right) \\
\leq & s^{n} \cdot 2 \delta+\frac{1}{1-s} \sup _{\omega} \max _{0 \leq t \leq a} r(\omega, t) .
\end{aligned}
$$

Since here $\omega$ is arbitrary, we get

$$
\gamma_{\omega}\left(s z^{-n}\right) \leq 2 \delta s^{n}+\frac{1}{1-s} \sup _{\omega} \max _{0 \leq t \leq a} r(t) .
$$

It follows that $\gamma_{\omega}(a) \rightarrow 0$ as $a \rightarrow 0$.

So $u$ is differentiable. Then from the definition of $v^{n}$ we have $v^{n} \in C^{0}$. Since $v^{n} \rightarrow v$ uniformly, $v \in C^{0}$. Since $D u=v, u \in C^{1}$. 
Next, we show $u \in C^{r}$. Knowing $u \in C^{1}$, by the construction of $v^{n}$, and using the partition of unity $\left\{h_{i}\right\}$, we have $v^{n} \in C^{1}$ and

$$
v_{j}^{n+1}\left(\theta^{T} \omega, \xi^{c u}\right)=\sum h_{i}\left[A+B v_{i}^{n}\left(\omega, x^{c u}\right)\right]\left[C+E v_{i}^{n}\left(\omega, x^{c u}\right)\right]^{-1} .
$$

Hence,

$$
\begin{aligned}
& D v_{j}^{n+1}\left(\theta^{T} \omega, \xi^{c u}\right)\left[C+E v_{i}\left(\omega, x^{c u}\right)\right] \\
& =\sum h_{i}\left[B D v_{i}^{n}\left(\omega, x^{c u}\right)\left(C+E v_{i}^{n}\left(\omega, x^{c u}\right)\right)^{-1}\right. \\
& \left.\quad-\left(A+B v_{i}^{n}\left(\omega, x^{c u}\right)\right)\left(C+E v_{i}^{n}\left(\omega, x^{c u}\right)\right)^{-1} E D v_{i}^{n}\left(\omega, x^{c u}\right)\left(C+E v_{i}^{n}\left(\omega, x^{c u}\right)\right)^{-1}\right] \\
& \quad+\left(\text { terms not involving derivatives of } v^{k} \text { or } v\right),
\end{aligned}
$$

which yields

$$
\begin{aligned}
& D v_{j}^{n+1}\left(\theta^{T} \omega, \xi^{c u}\right) \\
& =\sum h_{i}\left(B D v_{i}^{n}\left(\omega, x^{c u}\right)\left(C+E v_{i}^{n}\left(\omega, x^{c u}\right)\right)^{-1}\right. \\
& \left.\quad-\left(A+B v_{i}^{n}\left(\omega, x^{c u}\right)\right)\left(C+E v_{i}^{n}\left(\omega, x^{c u}\right)\right)^{-1} E D v_{i}^{n}\left(\omega, x^{c u}\right)\left(C+E v_{i}^{n}\left(\omega, x^{c u}\right)\right)^{-1}\right) \\
& \quad \times\left(C+E v_{i}\left(\omega, x^{c u}\right)\right)^{-1} \\
& \quad+\left(\text { terms not involving derivatives of } v^{k} \text { or } v\right) .
\end{aligned}
$$

Then, using Lemma 4.2 and arguments similar to those in Lemma 5.1 and 5.2 , one can show $D v^{n}$ is a Cauchy sequence. By the definition of $v^{n}, D v^{n} \in C^{0}$. Hence $D v$ exists and equals the limit of $D v^{n}$. So $v \in C^{1}$ and $u \in C^{2}$.

By using induction and Lemma 4.2, one can prove that $D^{k-1} v^{n}$ is a Cauchy sequence in $C^{0}$ and converges to $D^{k-1} v$ uniformly. So $v \in C^{k-1}$, i.e., $u \in C^{k}$. Thus, we have

\section{Proposition 5.3. $u \in C^{r}$.}

This completes the proof of the smoothness of the random unstable manifold $\tilde{\mathcal{W}}^{u}$. From the proof, we also obtain the following property, which will be used in the next section:

Proposition 5.4. Each $v_{i}$ is measurable in $\left(\omega, x^{c u}\right)$ jointly.

Proof. From the definition of the sequence $v_{n}$. Each $v^{n+1}$ is defined by $H\left(\omega, v^{n}\right)$ in a local chart. $H(\omega, v)$ has the following Carathéodory property: $H(\cdot, v)$ is measurable for any fixed $v$, and $H(\omega, \cdot)$ is $C^{r-1}$ for almost every $\omega \in \Omega$. So as long as $v^{0}$ is measurable in $\omega$ and $C^{r-1}$ in $x^{c u}$, each $v^{n}$ is measurable in $\omega$ and $C^{r-1}$ in $x^{c u}$. Since $v^{n}$ is measurable in $\omega$ and $C^{r-1}$ in $x^{c u}$, it is measurable in $\left(\omega, x^{c u}\right)$ jointly (see [CV]). Since $v^{0}$ is chosen to be 0 , all the above hold. Then the limit $v$ of $v^{n}$ is jointly measurable in $\left(\omega, x^{c u}\right)$.

\section{Measurability of the unstable manifold and its tangent SPaCE}

In this section, we prove that the unique random unstable manifold is a random set and the tangent space of the random unstable manifold is measurable.

Let $u=\{u(\omega): \omega \in \Omega\}$ be the unique fixed point of $G$ in $S_{\delta}$. For simplicity, we use $u(\omega)$ to denote both the section and the graph of the section.

Proposition 6.1. $u(\omega)$ is a random set. 
Proof. Recall

$$
\hat{U}_{k}^{i} \equiv\left(\sigma_{i} \times \tau_{i}^{u}\right)^{-1}\left(\mathcal{D}_{k}^{i} \times B(0, \epsilon)\right.
$$

and let

$$
\begin{gathered}
u^{0}(\omega)=\bigcup_{i=1}^{s} \hat{U}_{3}^{i}, \\
u^{1}(\omega)=\phi\left(T, \theta^{-T} \omega, u^{0}\left(\theta^{-T} \omega\right)\right) \cap V, \\
u^{n+1}(\omega)=\phi\left(T, \theta^{-T} \omega, u^{n}\left(\theta^{-T} \omega\right)\right) \cap V, \\
\cdots \\
u^{n}=\left\{u^{n}(\omega): \omega \in \Omega\right\} .
\end{gathered}
$$

Recall that $V$ is a tubular neighborhood of $M$ in $\mathbb{R}^{n}$ which is $C^{r}$ diffeomorphic to $\tilde{E}_{\epsilon}$. Since we identify $V$ with $\tilde{E}(\epsilon), u^{0}(\omega)$ can be viewed as a set or the zero section. Here, we view $u^{0}(\omega)$ as a set. We have $V \in \mathcal{B}\left(\mathbb{R}^{n}\right)$. Since $G$ is a uniform contraction on $S_{\delta}$, we have

$$
u^{n} \rightarrow u
$$

i.e.,

uniformly in $\omega \in \Omega$.

$$
u^{n}(\omega) \rightarrow u(\omega)
$$

We will show that all $u^{n}(\omega)$ are random sets. Let $\left\{m_{k}\right\}$ be dense in $\bigcup_{i=1}^{s} \hat{U}_{3}^{i}$. Define

and let

$$
y_{k}^{0}(\omega)=m_{k}
$$

$$
\begin{gathered}
y_{k}^{1}(\omega)=\phi\left(T, \theta^{-T} \omega, y_{k}^{0}\left(\theta^{-T} \omega\right)\right), \cdots, \\
y_{k}^{n+1}(\omega)=\phi\left(T, \theta^{-T} \omega, y_{k}^{n}\left(\theta^{-T} \omega\right)\right) .
\end{gathered}
$$

Since $\phi(t, \omega, m)$ is $\mathcal{B}(\mathbb{R}) \otimes \mathcal{F} \otimes \mathcal{B}\left(\mathbb{R}^{n}\right)$-measurable, we know that each $y_{k}^{n}(\omega)$ is $\mathcal{B}\left(\mathbb{R}^{n}\right)$-measurable. We also know that $\left\{y_{k}^{n}(\omega)\right\}_{k} \cap u^{n}(\omega)$ is dense in $u^{n}(\omega)$, since $\phi\left(-T, \theta^{T} \omega, \cdot\right)$ is continuous.

For any $x=x^{c u} \in \mathbb{R}^{n}$, define

$$
r_{x}^{n}(\omega)=\inf _{y \in u^{n}(\omega)}|x-y|
$$

then $r_{x}^{n}$ is a random variable. This is because for each fixed $a \in \mathbb{R}$,

$$
\begin{aligned}
& \left\{\omega: r_{x}^{n}(\omega) \geq a\right\} \\
& =\left\{\omega: \inf _{y \in u^{n}(\omega)}|x-y| \geq a\right\} \\
& =\bigcap_{k}\left(\left\{\omega\left|y_{k}^{n}(\omega) \in V,\right| x-y_{k}^{n}(\omega) \mid \geq a\right\} \cup\left\{\omega \mid y_{k}^{n}(\omega) \in V^{c}\right\}\right),
\end{aligned}
$$

which is $\mathcal{F}$-measurable because of the measurability of $y_{k}^{n}(\omega)$ and $V$. So we have proved $r_{x}^{n}(\omega)$ is a random variable. Since $n, x$ are arbitrary, $u^{n}(\omega)$ are random sets.

To show that $u(\omega)$ is a random set, let $x \in \mathbb{R}^{n}$ and define

$$
r_{x}(\omega)=\inf _{y \in u(\omega)}|x-y| .
$$

We want to show $r_{x}(\omega)$ is a random variable. Since

$$
u^{n}(\omega) \rightarrow u(\omega)
$$

uniformly for $\omega \in \Omega$ in $C^{0}$ norm, we have

$$
r_{x}^{n}(\omega) \rightarrow r_{x}(\omega)
$$


pointwise, which implies that $r_{x}(\omega)$ is a random variable, hence, $u_{\omega}$ is a random set. This completes the proof of the proposition.

To show the measurability of the tangent space $T \tilde{\mathcal{W}}^{u}$, we need to show

$$
T \tilde{\mathcal{W}}^{u}:(\omega, m) \in \tilde{\mathcal{W}}^{u} \rightarrow T_{(\omega, m)} \tilde{\mathcal{W}}^{u}
$$

is measurable. Since each $T_{(\omega, m)} \tilde{\mathcal{W}}^{u}$ is an $(n-k)$-dimensional subspace of $\mathbb{R}^{n}$, the map from $\tilde{\mathcal{W}}^{u}$ to the tangent space can be represented by

$$
\begin{gathered}
T \tilde{\mathcal{W}}^{u}: \tilde{\mathcal{W}}^{u}=\left\{(\omega, m): \omega \in \Omega, m \in \tilde{\mathcal{W}}^{u}(\omega)\right\} \rightarrow K_{n-k}, \\
(\omega, m) \mapsto T_{(\omega, m)} \tilde{\mathcal{W}}^{u} \in K_{n-k},
\end{gathered}
$$

where $K_{n-k}$ is the set of all $(n-k)$-dimensional subspaces of $\mathbb{R}^{n}$.

Consider the metric introduced by $[\mathrm{K}]$. Let $N_{1}, N_{2}$ be a linear subspace of $\mathbb{R}^{n}$ and let $S_{N_{1}}$ be the unit sphere in $N_{1}$. Define, for $N_{1} \neq\{0\} \neq N_{2}$,

$$
\begin{gathered}
d_{1}\left(N_{1}, N_{2}\right)=\sup _{x \in S_{N_{1}}} \operatorname{dist}\left(x, S_{N_{2}}\right), \\
d_{2}\left(N_{1}, N_{2}\right)=\max \left(d_{1}\left(N_{1}, N_{2}\right), d_{2}\left(N_{1}, N_{2}\right)\right) .
\end{gathered}
$$

Also define

$$
d_{2}(0, N)=d_{2}(N, 0)=1 .
$$

Then the set of all closed linear subspaces of $\mathbb{R}^{n}$ is a complete metric space with metric $d_{2}$ and $K_{n-k}$ is also a complete metric space with this metric. See also [LL].

Corresponding to the local chart $\mathcal{D}_{3}^{i} \times B(0, \epsilon) \times \mathbb{R}^{k}$, for each $\omega \in \Omega$, there is an open set $\hat{U}_{i}(\omega)$ on $\tilde{\mathcal{W}}^{u}(\omega)$. We denote

$$
\hat{U}_{i}:=\left\{(\omega, m): \omega \in \Omega, m \in \hat{U}_{i}(\omega)\right\} .
$$

Then

$$
\tilde{\mathcal{W}}^{u}=\hat{U}_{1} \cup \hat{U}_{2} \cup \cdots \cup \hat{U}_{s} .
$$

Each $\hat{U}_{i}$ is a measurable subset of $\tilde{\mathcal{W}}^{u}$.

In the local chart $\mathcal{D}_{3}^{i} \times B(0, \epsilon) \times \mathbb{R}^{k}, \hat{U}_{i}$ is constructed as a collection of Lipschitz functions $u_{i}\left(\omega, x^{c u}\right)$ over $\mathcal{D}_{3}^{i} \times B(0, \epsilon) . v_{i}\left(\omega, x^{c u}\right)$ is the derivative of $u_{i}(\omega, x)$ which has values in $L\left(\mathbb{R}^{n-k}, \mathbb{R}^{k}\right)$. We use $\hat{v}_{i}\left(\omega, x^{c u}\right)$ to denote the tangent space in the local chart. So $\hat{v}_{i}\left(\omega, x^{c u}\right)$ has values in $K_{n-k}$. By an elementary computation, for any $\omega \in \Omega, x^{c u} \in \mathcal{D}_{3}^{i} \times B(0, \epsilon)$, the $d_{2}$ distance between $\hat{v}_{i}\left(\omega, x^{c u}\right)$ and $\mathbb{R}^{n-k} \times\{0\}$ is at most $\sqrt{2-\frac{2}{\sqrt{1+\delta^{2}}}}$, which, for convenience, will be denoted by $\delta^{*}$. Then the range of $\hat{v}_{i}$ can be denoted by

$$
\overline{B_{d_{2}}\left(\mathbb{R}^{n-k} \times\{0\}, \delta^{*}\right)},
$$

the closed set containing all $(n-k)$-dimensional subspaces of $\mathbb{R}^{n}$, whose $d_{2}$ distance to $\mathbb{R}^{n-k} \times\{0\}$ are no more than $\delta^{*}$. Remember this set is in the chart $\mathcal{D}_{3}^{i} \times B(0, \epsilon) \times$ $\mathbb{R}^{k}$.

In order for $T \tilde{\mathcal{W}}^{u}$ to be a well-defined map, the range of all $T \tilde{\mathcal{W}}^{u} \mid \hat{U}_{i}$ should be in the same metric space. In other words, all values of $\hat{v}_{i}$ should be measured in a single chart. 
Suppose the coordinate change from $\mathcal{D}_{3}^{i} \times B(0, \epsilon) \times \mathbb{R}^{k}$ to $\mathcal{D}_{3}^{1} \times B(0, \epsilon) \times \mathbb{R}^{k}$ is represented by the invertible matrix $A_{i 1}$. Then in the chart $\mathcal{D}_{3}^{1} \times B(0, \epsilon) \times \mathbb{R}^{k}, \hat{v}$ is represented by $\left(A_{11} \hat{v}_{1}, \cdots, A_{s 1} \hat{v}_{s}\right)$. It has range

$$
\bigcup_{i=1}^{s} A_{i 1} \overline{B_{d_{2}}\left(\mathbb{R}^{n-k} \times\{0\}, \delta^{*}\right)} .
$$

To prove the measurability of $\hat{v}=T \tilde{\mathcal{W}}^{u}$, it is enough to prove the measurability of each $T \tilde{\mathcal{W}}^{u} \mid \hat{U}_{i}$ or, equivalently, the measurability of each $A_{i 1} \hat{v}_{i}$ as a function defined in the chart $\mathcal{D}_{3}^{i} \times B(0, \epsilon) \times \mathbb{R}^{k}$ and taking value in $K_{n-k}$ measured in the chart $\mathcal{D}_{3}^{1} \times B(0, \epsilon) \times \mathbb{R}^{k}$ :

$$
\Omega \times \mathcal{D}_{3}^{i} \times B(0, \epsilon) \rightarrow A_{i 1} \overline{B_{d_{2}}\left(\mathbb{R}^{n-k} \times\{0\}, \delta^{*}\right)} .
$$

Since $A_{i, 1}$ is an invertible matrix, it is a diffeomorphism from

$$
\overline{B_{d_{2}}\left(\mathbb{R}^{n-k} \times\{0\}, \delta^{*}\right)}
$$

to

$$
A_{i 1} \overline{B_{d_{2}}\left(\mathbb{R}^{n-k} \times\{0\}, \delta^{*}\right)} .
$$

So it suffices to prove the measurability of $\hat{v}_{i}$ as a function defined in the chart $\mathcal{D}_{3}^{i} \times B(0, \epsilon) \times \mathbb{R}^{k}$ and taking value in $K_{n-k}$ measured in the chart $\mathcal{D}_{3}^{i} \times B(0, \epsilon) \times \mathbb{R}^{k}$.

Proposition 6.2. $\hat{v}_{i}$ is measurable.

Proof. From Section 5 we know $v_{i}$ is measurable. We use the measurability of $v_{i}$ to prove the measurability of $\hat{v}_{i}$.

The operator norm of each $v_{i}(\omega, x)$ is at most $\delta$. Let $\overline{B(0, \delta)}$ be the closed ball centered at 0 with radius $\delta$ in the space $L\left(\mathbb{R}^{n-k}, \mathbb{R}^{k}\right)$. It induces a subset $K_{\delta}$ of $K_{n-k}$ :

$$
K_{\delta}:=\left\{(a, \tilde{L} a): a \in R^{n-k}, \tilde{L} \in \overline{B(0, \delta)}\right\} .
$$

We also have the representation

$$
K_{\delta}=\overline{B_{d_{2}}\left(\mathbb{R}^{n-k} \times\{0\}, \delta^{*}\right)} .
$$

This is because for any $\tilde{L} \in \overline{B(0, \delta)}$,

$$
d_{2}\left(\mathbb{R}^{n-k} \times\{0\},(I \times \tilde{L})\left(\mathbb{R}^{n-k}\right)\right)=\sqrt{2-\frac{2}{\sqrt{1+\|\tilde{L}\|^{2}}}},
$$

which is no more than $\delta^{*}=\sqrt{2-\frac{2}{\sqrt{1+\delta^{2}}}}$. On the other hand, for any $0<\delta_{1} \leq \delta$, and $J$ an $(n-k)$-dimensional subspace of $\mathbb{R}^{n}$ whose $d_{2}$ distance to $\mathbb{R}^{n-k} \times\{0\}$ is $\delta_{1}$, there exists an $\tilde{L}$ such that $\|\tilde{L}\|=\delta_{1}$ and $\mathbb{R}^{n-k} \times \tilde{L}\left(\mathbb{R}^{n-k}\right)=J$. So we have

$$
K_{\delta}=\overline{B_{d_{2}}\left(\mathbb{R}^{n-k} \times\{0\}, \delta^{*}\right)} .
$$

Define a metric $d_{3}$ on $K_{\delta}$ by

$$
d_{3}\left(\mathbb{R}^{n-k} \times L_{1}\left(\mathbb{R}^{n-k}\right), \mathbb{R}^{n-k} \times L_{2}\left(\mathbb{R}^{n-k}\right)\right):=\left\|L_{1}-L_{2}\right\|_{L\left(\mathbb{R}^{n-k}, \mathbb{R}^{k}\right)} .
$$

This makes $K_{\delta}$ into a metric space, and obviously we have

$$
K_{\delta}=\overline{B_{d_{3}}\left(\mathbb{R}^{n-k} \times\{0\}, \delta\right)} .
$$


Lemma 6.1. $d_{2}$ and $d_{3}$ generate the same topology on $K_{\delta}$.

Proof. Let $D_{n} \subset K_{\delta}$. It is obvious that $d_{2}\left(D_{n}, D_{0}\right) \rightarrow 0$ if and only if $d_{3}\left(D_{n}, D_{0}\right) \rightarrow$ 0 .

We use the metric $d_{3}$ on $K_{\delta}$. This makes $K_{\delta}$ isomorphic to $\overline{B(0, \delta)}$. So the measurability of $\hat{v}_{i}$ as a function to $K_{\delta}$ is equivalent to the measurability of $v_{i}$ as a function to $\overline{B(0, \delta)}$. By Proposition [5.4, $v_{i}$ is measurable, so $\hat{v}_{i}$ is measurable.

From the discussion before Proposition 6.2, we have:

Proposition 6.3. The tangent space of the random unstable manifold $\tilde{\mathcal{W}}^{u}$ is measurable.

\section{EXISTENCE OF THE RANDOM STABLE MANIFOLD}

Since the random flow $\phi(t, \omega)$ is invertible, by reversing the time and applying the results on unstable manifold in Sections 4, 5, and 6 , we have

Proposition 7.1. There exists a unique $C^{r}$ random stable manifold $\tilde{\mathcal{W}}^{s}(\omega)$ in the neighborhood $V$ of $\mathcal{M}$. Moreover, it is a random set and its tangent space is measurable.

Taking the intersection of $\tilde{\mathcal{W}}^{u}(\omega)$ and $\tilde{\mathcal{W}}^{s}(\omega)$, we get an invariant manifold $\tilde{\mathcal{M}}(\omega)$ of the random flow $\phi(t, \omega)$. From the proof of the existence of $\tilde{\mathcal{W}}^{s}(\omega)$ and $\tilde{\mathcal{W}}^{u}(\omega)$ we can find this intersection by taking a measurable $m$-dimensional random manifold $\mathcal{M}^{0}(\omega)$ on the random unstable manifold as a graph over $\mathcal{M}$ and mapping it under the inverse flow. For example, we can take the random set which corresponds to $u_{i}\left(\omega, x^{c u}\right)=u_{i}\left(\omega, x^{c}, 0\right)$ in a local chart. Iterates of this random set approach the random stable manifold uniformly under the inverse random flow. On the other hand, the random set stays in the random unstable manifold. So it converges to the intersection of the random stable and unstable manifolds. In other words, the invariant manifold of intersection is the limit of $\mathcal{M}^{0}(\omega)$ under the inverse random flow. Since we have the measurability and smoothness of $u_{i}\left(\omega, x^{c}, 0\right)$ in local charts, we know $\mathcal{M}^{0}(\omega)$ is measurable and smooth, and also since the derivatives (tangent spaces) converge uniformly, then the limit $\tilde{\mathcal{M}}(\omega)$ is also measurable and smooth.

$\tilde{\mathcal{M}}(\omega)$ is obviously $C^{r}$ diffeomorphic to $\mathcal{M}$ for each $\omega \in \Omega$ because it is the graph of a $C^{r}$ section over the tangent bundle of $\mathcal{M}$.

The measurability of the tangent space of $\tilde{\mathcal{M}}(\omega)$ is also proved from the measurability and smoothness of the tangent space of $\mathcal{M}^{0}(\omega)$. From the measurability and smoothness of the tangent space of $\mathcal{M}^{0}(\omega)$, we get the measurability of the derivative map of $\mathcal{M}^{0}(\omega, m)$ in local charts using the Carathéodory property as we did in Proposition 5.4. Then from uniform convergence of the tangent spaces, we get the measurability of the derivative map of $\mathcal{M}(\omega)$ in local charts. Applying the method of proving the measurability of the tangent space of the unstable manifold, which shows the equivalence between the measurability of the tangent space and the measurability of the tangent map, we obtain the measurability of the tangent space of the random invariant manifold $\mathcal{M}(\omega)$.

Summarizing the above, we get

Proposition 7.2. There exists a unique $C^{r}$ random invariant manifold $\tilde{\mathcal{M}}(\omega)$ in a neighborhood of $\mathcal{M}$. For each fixed $\omega \in \Omega, \tilde{\mathcal{M}}(\omega)$ is $C^{r}$ diffeomorphic to $\mathcal{M}$. Moreover, it is a random set and its tangent space is measurable. 


\section{Persistence of normal hyperbolicity}

In this section we show that $\tilde{\mathcal{M}}$ is normally hyperbolic. We prove the following proposition:

Proposition 8.1. For each $x \in \tilde{\mathcal{M}}(\omega)$ there exists a splitting

$$
\mathbb{R}^{n}=E^{u}(\omega, x) \oplus E^{c}(\omega, x) \oplus E^{s}(\omega, x)
$$

of closed subspaces with associated projections $\Pi^{u}(\omega, x), \Pi^{c}(\omega, x)$, and $\Pi^{s}(\omega, x)$ such that

(i) The splitting is invariant:

$$
D_{x} \phi(t, \omega)(x) E^{i}(\omega, x)=E^{i}\left(\theta_{t} \omega, \phi(t, \omega)(x)\right), \quad \text { for } i=u, c, s .
$$

(ii) $\left.D_{x} \phi(t, \omega)(x)\right|_{E^{i}(\omega, x)}: E^{i}(\omega, x) \rightarrow E^{i}\left(\theta_{t} \omega, \phi(t, \omega)(x)\right)$ is an isomorphism for $i=u, c, s . E^{c}(\omega, x)$ is the tangent space of $\mathcal{M}(\omega)$ at $x$.

(iii) There are $(\theta, \phi)$-invariant random variables $\alpha, \beta: \mathcal{M} \rightarrow(0, \infty), 0<\alpha<\beta$, and a tempered random variable $K(\omega, x): \mathcal{M} \rightarrow[1, \infty)$ such that

$$
\begin{aligned}
& \left\|D_{x} \phi(t, \omega)(x) \Pi^{s}(\omega, x)\right\| \leq K(\omega, x) e^{-\beta(\omega, x) t} \quad \text { for } t \geq 0, \\
& \left\|D_{x} \phi(t, \omega)(x) \Pi^{u}(\omega, x)\right\| \leq K(\omega, x) e^{\beta(\omega, x) t} \quad \text { for } t \leq 0, \\
& \left\|D_{x} \phi(t, \omega)(x) \Pi^{c}(\omega, x)\right\| \leq K(\omega, x) e^{\alpha(\omega, x)|t|} \quad \text { for }-\infty<t<\infty .
\end{aligned}
$$

Moreover, $E^{i}(\omega, x)$ are measurable in $(\omega, x)$ and $C^{r-1}$ in $x$.

We prove the proposition by several lemmas.

Lemma 8.1. For any $\omega \in \Omega$, there are subbundles $\tilde{E}^{s}(\omega)$ and $\tilde{E}^{u}(\omega)$ of $T \mathbb{R}^{n} \mid \tilde{\mathcal{M}}(\omega)$, which are uniformly close to $\tilde{E}^{s}$ and $\tilde{E}^{u}$ respectively, such that $\tilde{E}^{s}(\omega)$ is complementary to $T \tilde{\mathcal{M}}(\omega)$ in $T \tilde{\mathcal{W}}^{s}(\omega) \mid \tilde{\mathcal{M}}(\omega)$ and $\tilde{E}^{u}$ is complementary to $T \tilde{\mathcal{M}}(\omega)$ in $T \tilde{\mathcal{W}}^{s}(\omega) \mid \tilde{\mathcal{M}}(\omega)$.

Proof. We first note that there exists an unstable manifold $\mathcal{W}^{u}$ of $\mathcal{M}$ under the deterministic flow $\psi(t)$. Both $\mathcal{W}^{u}$ and $\tilde{\mathcal{W}}^{u}(\omega)$ are constructed as sections over the same bundle. Since $\phi(t, \omega)$ and $\psi(t)$ are uniformly $C^{1}$ close, $\mathcal{W}^{u}$ and $\tilde{\mathcal{W}}^{u}(\omega)$ are $C^{1}$ close and diffeomorphic to each other. We denote the diffeomorphism between them by $u(\omega)$. Then $u(\omega)$ is $C^{1}$ close to the identity map. Taking the image of $\tilde{E}^{u}$ under the map $D u(\omega)$ to get $\tilde{E}^{u}(\omega)$, then $\tilde{E}^{u}(\omega)$ is uniformly close to $\tilde{E}^{u}$.

Similarly, we have that $\tilde{E}^{s}(\omega)$ is uniformly close to $\tilde{E}^{s}$.

Since $\tilde{E}^{i}$ is arbitrarily close to $E^{i}$ for $i=s, u$, we have that $\tilde{E}^{i}(\omega)$ is uniformly close to $E^{i}$. Since there is no invariance condition on $\tilde{E}^{i}(\omega)$, we can modify $\tilde{E}^{i}(\omega, m)$ such that it is $C^{r}$ in $m$ for each fixed $\omega \in \Omega$ and still stays close to $E^{i}$. This is from W]. In any case, $\tilde{E}^{i}(\omega, m)$ is not necessarily measurable.

Define $\tilde{E}^{c}(\omega, m) \equiv T \tilde{\mathcal{M}}(\omega)$, and for $i=s, u, c, \tilde{\Pi}^{i} \equiv \tilde{\Pi}^{i}(\omega, m)$ the projections onto $\tilde{E}^{i}(\omega, m)$. Recall that $0<\alpha<\beta$ are constants associated with the normal hyperbolicity of $\mathcal{M}$ with respect to $\psi(t)$.

Lemma 8.2. (1) There exist positive constants $0<a<1$ and $c_{1}$ such that

$$
\left\|\tilde{\Pi}^{s} D \phi\left(t, \theta^{-t} \omega\right)(\phi(-t, \omega)(m)) \mid \tilde{E}^{s}\left(\theta^{-t} \omega\right)\right\|<c_{1} a^{t}
$$


for all $m \in \tilde{\mathcal{M}}(\omega)$ and $t \geq 0$, and

$$
\left\|\tilde{\Pi}^{u} D \phi\left(t, \theta^{-t} \omega\right)(\phi(-t, \omega)(m)) \mid \tilde{E}^{u}\left(\theta^{-t} \omega\right)\right\|<c_{1} a^{t}
$$

for all $m \in \tilde{\mathcal{M}}(\omega)$ and $t \leq 0$.

(2) If $\alpha<r \beta$, there exist $c_{2}>0$ and $r^{\prime}>r$ such that

$$
\begin{aligned}
& \left\|\tilde{\Pi}^{s} D \phi\left(t, \theta^{-t} \omega\right)(\phi(-t, \omega)(m))\left|\tilde{E}^{s}\left(\theta^{-t} \omega\right)\|\| D \phi(-t, \omega)(m)\right| \tilde{E}^{c}(\omega)\right\|^{r^{\prime}}<c_{2} \\
& \quad \text { for all } m \in \tilde{\mathcal{M}}(\omega) \text { and } t \geq 0 \text {, and } \\
& \left\|\tilde{\Pi}^{u} D \phi\left(t, \theta^{-t} \omega\right) \phi(-t, \omega)(m)\left|\tilde{E}^{u}\left(\theta^{-t} \omega\right)\|\| D \phi(-t, \omega)(m)\right| \tilde{E}^{c}(\omega)\right\|^{r^{\prime}}<c_{2} \\
& \quad \text { for all } m \in \tilde{\mathcal{M}}(\omega) \text { and } t \leq 0 \text {. }
\end{aligned}
$$

(3) If $\alpha<r \beta$, there exist $c_{3}>0$ and $r^{\prime}>r$ such that

$$
\begin{aligned}
& \left\|\tilde{\Pi}^{s} D \phi\left(t, \theta^{-t} \omega\right) \phi(-t, \omega)(m)\left|\tilde{E}^{s}\left(\theta^{-t} \omega\right)\|\| D \phi(-t, \omega)(m)\right| \tilde{E}^{c}(\omega)\right\| \\
& \left\|D \phi\left(t, \theta^{-t} \omega\right) \phi(-t, \omega)(m) \mid \tilde{E}^{c}\left(\theta^{-t} \omega\right)\right\|^{r^{\prime}-1}<c_{3}
\end{aligned}
$$

for all $m \in \tilde{\mathcal{M}}(\omega)$ and $t \geq 0$, and

$$
\begin{aligned}
& \qquad\left\|\tilde{\Pi}^{u} D \phi\left(t, \theta^{-t} \omega\right) \phi(-t, \omega)(m)\left|\tilde{E}^{u}\left(\theta^{-t} \omega\right)\|\| D \phi(-t, \omega)(m)\right| \tilde{E}^{c}(\omega)\right\| \\
& \left\|D \phi\left(t, \theta^{-t} \omega\right) \phi(-t, \omega)(m)\left|\tilde{E}^{c}\left(\theta^{-t} \omega\right) \|\right|^{r^{\prime}-1}<c_{3}\right. \\
& \text { for all } m \in \tilde{\mathcal{M}}(\omega) \text { and } t \leq 0 .
\end{aligned}
$$

Proof. We prove this lemma using an idea extended from [F1].

We first prove (1). Since

$$
\left\|D \psi(t) \psi(-t)(m) \mid E^{s}\right\| \rightarrow 0 \text { as } t \rightarrow \infty
$$

for all $m \in \mathcal{M}$, for $m \in \mathcal{M}$ there exists $T(m)>0$ such that

$$
\left\|D \psi(T(m)) \psi(-T(m))(m) \mid E^{s}\right\|<1 \text {. }
$$

Hence, there exists a neighborhood $U(m)$ of $m$ in $\mathcal{M}$ such that for $m^{\prime} \in U(m)$,

$$
\left\|D \psi(T(m)) \psi(-T(m))\left(m^{\prime}\right) \mid E^{s}\right\|<1 .
$$

Since $\mathcal{M}$ is compact, we may choose finitely many points $m_{1}, m_{2}, \cdots, m_{N}$ such that

$$
\mathcal{M} \subset U\left(m_{1}\right) \cup U\left(m_{2}\right) \cup \cdots \cup U\left(m_{N}\right) .
$$

Choose $0<a<1$ such that

$$
\left\|D \psi\left(T\left(m_{i}\right)\right) \psi\left(-T\left(m_{i}\right)\right)\left(m^{\prime}\right) \mid E^{s}\right\|<a^{T\left(m_{i}\right)}
$$

for $m^{\prime} \in U\left(m_{i}\right)$. Since $\phi(t, \omega)$ and $\psi(t)$ are uniformly close and $\tilde{\mathcal{M}}(\omega)$ and $\mathcal{M}$ are uniformly close, we have

$$
\left\|\tilde{\Pi}^{s} D \phi\left(T\left(m_{i}\right), \theta^{-T\left(m_{i}\right)} \omega\right) \phi\left(-T\left(m_{i}\right), \omega\right)\left(u\left(\omega, m^{\prime}\right)\right) \mid \tilde{E}^{s}\left(\theta^{-T\left(m_{i}\right)} \omega\right)\right\|<a^{T\left(m_{i}\right)}
$$

for $m^{\prime} \in U\left(m_{i}\right)$. Take $U\left(\omega, m_{i}\right)=u\left(\omega, U\left(m_{i}\right)\right)$. Then

$$
\tilde{\mathcal{M}}(\omega) \subset U\left(\omega, m_{1}\right) \cup U\left(\omega, m_{2}\right) \cup \cdots \cup U\left(\omega, m_{N}\right)
$$

and

$$
\left\|\tilde{\Pi}^{s} D \phi\left(T\left(m_{i}\right), \theta^{-T\left(m_{i}\right)} \omega\right) \phi\left(-T\left(m_{i}\right), \omega\right)(\tilde{m}) \mid \tilde{E}^{s}\left(\theta^{-T\left(m_{i}\right)} \omega\right)\right\|<a^{T\left(m_{i}\right)}
$$

for all $\omega \in \Omega$ and $\tilde{m} \in U\left(\omega, m_{i}\right)$.

Fix an arbitrary $\omega \in \Omega$ and let $m \in \tilde{\mathcal{M}}(\omega)$ be given. Choose a sequence of integers $i(1), i(2), \cdots$, as follows. Choose $i(1)$ such that $m \in U\left(\omega, m_{i(1)}\right)$. If $i(1), i(2), \cdots, i(j)$ have been chosen, let $\tau(j)=T\left(m_{i(1)}\right)+\cdots+T\left(m_{i(j)}\right)$. Choose 
$i(j+1)$ such that $\phi(-\tau(j), \omega)(m) \in U\left(\theta^{-\tau(j)} \omega, m_{i(j+1)}\right)$. Letting $t>0$, it is possible to write $t=\tau(j)+r$ for some $j$ and $0 \leq r<\max T\left(m_{i}\right)$. Then

$$
\begin{aligned}
& \left\|\tilde{\Pi}^{s} D \phi\left(t, \theta^{-t} \omega\right) \phi(-t, \omega)(m) \mid \tilde{E}^{s}\left(\theta^{-t} \omega\right)\right\| \\
= & \| \tilde{\Pi}^{s} D \phi\left(T\left(m_{i(1)}\right), \theta^{-\tau(1)} \omega\right) \phi\left(-T\left(m_{i(1)}\right), \omega\right)(m) \mid \tilde{E}^{s}\left(\theta^{-\tau(1)} \omega\right) \\
& \tilde{\Pi}^{s} D \phi\left(T\left(m_{i(2)}\right), \theta^{-\tau(2)} \omega\right) \phi\left(-T\left(m_{i(2)}\right), \theta^{-\tau(1)} \omega\right) \phi(-\tau(1), \omega)(m) \mid \tilde{E}^{s}\left(\theta^{-\tau(2)} \omega\right) \\
& \tilde{\Pi}^{s} D \phi\left(T\left(m_{i(3)}\right), \theta^{-\tau(3)} \omega\right) \phi\left(-T\left(m_{i(3)}\right), \theta^{-\tau(2)} \omega\right) \phi(-\tau(2), \omega)(m) \mid \tilde{E}^{s}\left(\theta^{-\tau(3)} \omega\right) \\
& \ldots \\
& \tilde{\Pi}^{s} D \phi\left(r, \theta^{-t} \omega\right) \phi\left(-r, \theta^{-\tau(j)} \omega\right) \phi(-\tau(j), \omega)(m) \mid \tilde{E}^{s}\left(\theta^{-t} \omega\right) \| \\
\leq & a^{T\left(m_{1}\right)} \cdot a^{T\left(m_{2}\right)} \cdots a^{T\left(m_{j}\right)} \cdot a^{r} \\
& . \frac{\left\|\tilde{\Pi}^{s} D \phi\left(r, \theta^{-t} \omega\right) \phi\left(-r, \theta^{-\tau(j)} \omega\right) \phi(-\tau(j), \omega)(m) \mid \tilde{E}^{s}\left(\theta^{-t} \omega\right)\right\|}{a^{r}} \\
\leq & c a^{t},
\end{aligned}
$$

where

$$
c=\sup \frac{\left\|\tilde{\Pi}^{s} D \phi\left(r, \theta^{-t} \omega\right) \phi\left(-r, \theta^{-\tau(j)} \omega\right) \phi(-\tau(j), \omega)(m) \mid \tilde{E}^{s}\left(\theta^{-t} \omega\right)\right\|}{a^{r}}
$$

and the supremum is taken over all $\omega \in \Omega, m \in \tilde{\mathcal{M}}(\omega)$ and $0 \leq r<\max T\left(m_{i}\right)$. Equivalently,

$$
c=\sup \frac{\left\|\tilde{\Pi}^{s} D \phi\left(r, \theta^{-r} \omega^{\prime}\right) \phi\left(-r, \omega^{\prime}\right)\left(m^{\prime}\right) \mid \tilde{E}^{s}\left(\theta^{-r} \omega^{\prime}\right)\right\|}{a^{r}},
$$

and the supremum is taken over all $\omega^{\prime} \in \Omega, m^{\prime} \in \tilde{\mathcal{M}}\left(\omega^{\prime}\right)$ and $0 \leq r<\max T\left(m_{i}\right)$.

Since (2) and (3) follow similar arguments, we omit the details.

Lemma 8.3. There exist unique subbundles $E^{s}(\omega)$ and $E^{u}(\omega)$ of $T \mathbb{R}^{n} \mid \tilde{\mathcal{M}}(\omega)$ such that $E^{s}(\omega)$ is complementary to $T \tilde{\mathcal{M}}(\omega)$ in $T \tilde{\mathcal{W}}^{s}(\omega) \mid \tilde{\mathcal{M}}(\omega)$ and $E^{u}(\omega)$ is complementary to $T \tilde{\mathcal{M}}(\omega)$ in $T \tilde{\mathcal{W}}^{u}(\omega) \mid \tilde{\mathcal{M}}(\omega)$. Moreover, $E^{i}(\omega)(i=s, u)$ is $C^{r-1}$ and invariant under $D \phi(t, \omega)$ for any $t \in \mathbb{R}$ and $\omega \in \Omega$.

Proof. We will show that for some $K$ to be determined later, there exists a unique random subbundle $E^{u}(\omega)$ of $T \tilde{\mathcal{W}}^{s}(\omega) \mid \tilde{\mathcal{M}}(\omega)$ that is invariant under $D \phi(K, \omega)$. This will be sufficient to conclude the invariance under $D \phi(t, \omega)$ for any $t \in \mathbb{R}$. This is because once we proved the first, we know that $D \phi(t, \omega)\left(E^{u}(\omega)\right)$ is also invariant under $D \phi(K, \omega)$. Then by the uniqueness we are done.

To start, we notice that any bundle complementary to $T \tilde{\mathcal{M}}(\omega)$ in $T \tilde{\mathcal{W}}^{u}(\omega) \mid \tilde{\mathcal{M}}(\omega)$ is the graph of a family of linear maps

$$
h(\omega, m): \tilde{E}^{u}(\omega, m) \rightarrow T \tilde{\mathcal{M}}(\omega, m),
$$

and the bundle is invariant under $D \phi(K, \omega)$ if and only if

$$
D \phi\left(K, \theta^{-K} \omega\right) h\left(\theta^{-K} \omega, \phi(-K, \omega, m)\right)=h(\omega, m)
$$

for all $\omega \in \Omega$ and $m \in \tilde{\mathcal{M}}(\omega)$. Equivalently,

$$
\begin{aligned}
& h(\omega, m) \tilde{\Pi}^{u} D \phi\left(K, \theta^{-K} \omega, \phi(-K, \omega, m)\right) \cdot\left(\xi^{c u}+h\left(\theta^{-K} \omega, \phi(-K, \omega, m)\right)\right) \xi^{c u} \\
= & \tilde{\Pi}^{c} D \phi\left(K, \theta^{-K} \omega, \phi(-K, \omega, m)\right) \cdot\left(\xi+h\left(\theta^{-K} \omega, \phi(-K, \omega, m)\right)\right) \xi
\end{aligned}
$$


for all $\xi \in \tilde{E}^{u}\left(\theta^{-K} \omega, \phi(-K, \omega, m)\right)$, for $\omega \in \Omega, m \in \tilde{\mathcal{M}}(\omega)$. Since $T \tilde{\mathcal{M}}(\omega)$ is invariant, we have

$$
\tilde{\Pi}^{u} D \phi\left(K, \theta^{-K} \omega, \phi(-K \omega, m)\right) h\left(\theta^{-K} \omega, \phi(-K, \omega, m)\right)=0 .
$$

Suppressing $\xi$, we can write the functional equation for $h$ as

$$
\begin{aligned}
& h(\omega, m) \circ \tilde{\Pi}^{u} D \phi\left(K, \theta^{-K} \omega, \phi(-K, \omega, m)\right) \mid \tilde{E}^{u}\left(\theta^{-K} \omega, \phi(-K, \omega, m)\right) \\
= & \tilde{\Pi}^{c} D \phi\left(K, \theta^{-K} \omega, \phi(-K, \omega, m)\right) \mid \tilde{E}^{u}\left(\theta^{-K} \omega, \phi(-K, \omega, m)\right) \\
& +\tilde{\Pi}^{c} D \phi\left(K, \theta^{-K} \omega, \phi(-K, \omega, m)\right) \\
& \circ h\left(\theta^{-K} \omega, \phi(-K, \omega, m)\right) \mid \tilde{E}^{u}\left(\theta^{-K} \omega, \phi(-K, \omega, m)\right) .
\end{aligned}
$$

We have

$\left[\tilde{\Pi}^{u} D \phi\left(K, \theta^{-K} \omega, \phi(-K, \omega, m)\right) \mid \tilde{E}^{u}\left(\theta^{-K} \omega, \phi(-K, \omega, m)\right)\right]^{-1}=\tilde{\Pi}^{u} D \phi(-K, \omega, m) \mid \tilde{E}^{u}$.

Hence,

$$
\begin{aligned}
& h(\omega, m) \\
= & \tilde{\Pi}^{c} D \phi\left(K, \theta^{-K} \omega, \phi(-K, \omega, m)\right) \circ \tilde{\Pi}^{u} D \phi(-K, \omega, m) \mid \tilde{E}^{u} \\
& +\tilde{\Pi}^{c} D \phi\left(K, \theta^{-K} \omega, \phi(-K, \omega, m)\right) \circ h\left(\theta^{-K} \omega, \phi(-K, \omega, m)\right) \\
& \circ \tilde{\Pi}^{u} D \phi(-K, \omega, m) \mid \tilde{E}^{u} .
\end{aligned}
$$

This is a linear functional equation for $h$. We want to use the contraction mapping theorem to show it has a unique fixed point.

By Proposition 8.2 (1) and (2), we get

$$
\begin{gathered}
\left\|D\left(\phi \mid \tilde{\mathcal{M}}\left(\theta^{-K} \omega\right)\right)\left(K, \theta^{-K} \omega, \phi(-K, \omega, m)\right)\right\|\left\|\tilde{\Pi}^{u} D \phi(-K, \omega, m) \mid \tilde{E}^{u}\right\| \leq \frac{1}{4} \\
\left\|D \phi\left(K, \theta^{-K} \omega, \phi(-K, \omega, m)\right)\left|\tilde{E}^{c}\left(\theta^{-K} \omega\right)\|\| D \phi(-K, \omega, m)\right| \tilde{E}^{c}(\omega)\right\|^{k} \\
\left\|\tilde{\Pi}^{u} D \phi(-K, \omega, m) \mid \tilde{E}^{u}\right\| \leq \frac{1}{4}
\end{gathered}
$$

for $1 \leq k \leq r-1$.

Define a space $S$ whose element $h \in S$ is of the form

$$
h=\{h(\omega, m) \mid \omega \in \Omega, m \in \tilde{\mathcal{M}}(\omega)\} .
$$

Define the norm $\|\cdot\|_{S}$ by

$$
\|h\|_{S}:=\sup _{\omega} \max _{m \in \tilde{\mathcal{M}}(\omega)}\|h(\omega, m)\|_{L\left(\tilde{E}^{u}(\omega, m), T \tilde{\mathcal{M}}(\omega, m)\right)} .
$$

Under this norm, $S$ is a complete metric space. Let

$$
h^{0}(\omega, m) \equiv 0 \in L\left(\tilde{E}^{u}(\omega, m), T \tilde{\mathcal{M}}(\omega, m)\right),
$$

and define

$$
\begin{aligned}
& h^{n+1}(\omega, m) \\
= & \tilde{\Pi}^{c} D \phi\left(K, \theta^{-K} \omega, \phi(-K, \omega, m)\right) \circ \tilde{\Pi}^{u} D \phi(-K, \omega, m) \mid \tilde{E}^{u} \\
& +\tilde{\Pi}^{c} D \phi\left(K, \theta^{-K} \omega, \phi(-K, \omega, m)\right) \circ h^{n}\left(\theta^{-K} \omega, \phi(-K, \omega, m)\right) \\
& \circ \tilde{\Pi}^{u} D \phi(-K, \omega, m) \mid \tilde{E}^{u} .
\end{aligned}
$$


Then for all $n \geq 0$ and for any fixed $\omega, h^{n}(\omega, m)$ is $C^{r-1}$ in $m$. By (8.4), $h^{n}$ is a Cauchy sequence. Suppose $h$ is the unique limit of $h^{n}$. Then $h$ satisfies

$$
\begin{aligned}
& h(\omega, m) \\
= & \tilde{\Pi}^{c} D \phi\left(K, \theta^{-K} \omega, \phi(-K, \omega, m)\right) \circ \tilde{\Pi}^{u} D \phi(-K, \omega, m) \mid \tilde{E}^{u} \\
& +\tilde{\Pi}^{c} D \phi\left(K, \theta^{-K} \omega, \phi(-K, \omega, m)\right) \circ h\left(\theta^{-K} \omega, \phi(-K, \omega, m)\right) \\
& \circ \tilde{\Pi}^{u} D \phi(-K, \omega, m) \mid \tilde{E}^{u},
\end{aligned}
$$

so $h(\omega, m)$ represents the unique invariant bundle $E(\omega, m)$. Since $h^{n}(\omega, m) \rightarrow$ $h(\omega, m)$ uniformly, $h(\omega, m)$ is $C^{0}$ in $m$.

By (8.5), $D_{m}^{k} h^{n}(\omega, m)$ for $k \leq r-1$ is a Cauchy sequence in the corresponding space. So $D_{m}^{k} h^{n}(\omega, m)$ converges uniformly as $n$ goes to $\infty$. Therefore, $D_{m}^{k} h(\omega, m)$ exists and equals the limit of $D_{m}^{k} h^{n}(\omega, m)$, which means $h(\omega, m)$ is $C^{r-1}$ in $m$ for any fixed $\omega$. Hence, we obtain a unique $C^{r-1}$ invariant bundle $E^{u}(\omega)$. Similarly, we have a unique $C^{r-1}$ invariant bundle $E^{s}(\omega)$.

From now on, we use $E^{s}(\omega)$ and $E^{u}(\omega)$ to denote the unique invariant bundle of Lemma 8.3. Since $\psi(t)$ and $\phi(t, \omega)$ are uniformly close, and $\mathcal{M}$ and $\tilde{\mathcal{M}}(\omega)$ is uniformly close, we conclude that $E(\omega)$ is uniformly close to $E$. Since $\mathcal{M}$ is compact, the angle between $E(m)$ and $T_{m} \mathcal{M}$ is uniformly bounded away from 0 . So we also have that the angle between $E(\omega, m)$ and $T_{m} \tilde{\mathcal{M}}(\omega)$ is uniformly bounded away from 0 . So $\left\|\Pi^{s}\right\|,\left\|\Pi^{u}\right\|, \frac{1}{\left\|\Pi^{s}\right\|}$ and $\frac{1}{\left\|\Pi^{u}\right\|}$ are bounded. Suppose they are all bounded by $c_{4}>0$.

Lemma 8.4. (8.1), (8.2) and (8.3) are satisfied for $E^{s}(\omega), E^{u}(\omega)$ and $E^{c}(\omega)=$ $T \tilde{\mathcal{M}}(\omega)$.

Proof. For any $\nu \in T \tilde{\mathcal{W}}^{s}(\omega) \mid \tilde{\mathcal{M}}(\omega)$, we have

$$
\nu-\Pi^{s} \nu \in T \tilde{\mathcal{M}}(\omega) \text {. }
$$

So $\tilde{\Pi}^{s} \Pi^{s}=\tilde{\Pi}^{s}$. Similarly, $\Pi^{s} \tilde{\Pi}^{s}=\Pi^{s}$.

For each fixed $\omega \in \Omega, m \in \tilde{\mathcal{M}}(\omega)$ and $\nu \in E^{s}(\omega, m)$, let $\tilde{\nu}=\tilde{\Pi}^{s} \nu$. Then $\tilde{\nu}-\nu \in T \tilde{\mathcal{M}}(m)$ and

$$
\begin{aligned}
& c_{4}^{-1}\left|\tilde{\Pi}^{s} D \phi(-t, \omega)(\tilde{\nu})\right| \\
= & c_{4}^{-1}\left|\tilde{\Pi}^{s} D \phi(-t, \omega)\left(\tilde{\Pi}^{s} \nu\right)\right|=c_{4}^{-1}\left|\tilde{\Pi}^{s} D \phi(-t, \omega)(\nu)\right| \leq\left|\Pi^{s} \tilde{\Pi}^{s} D \phi(-t, \omega)(\nu)\right| \\
= & \left|\Pi^{s} D \phi(-t, \omega)(\nu)\right| \leq c_{4}\left|\tilde{\Pi}^{s} \Pi^{s} D \phi(-t, \omega)(\nu)\right|=c_{4}\left|\tilde{\Pi}^{s} D \phi(-t, \omega)(\nu)\right| \\
= & c_{4}\left|\tilde{\Pi}^{s} D \phi(-t, \omega)\left(\tilde{\Pi}^{s} \nu\right)\right|=c_{4}\left|\tilde{\Pi}^{s} D \phi(-t, \omega)(\tilde{\nu})\right|,
\end{aligned}
$$

which gives us

$$
c_{4}^{-1}\left|\tilde{\Pi}^{s} D \phi(-t, \omega)(\tilde{\nu})\right| \leq\left|\Pi^{s} D \phi(-t, \omega)(\nu)\right| \leq c_{4}\left|\tilde{\Pi}^{s} D \phi(-t, \omega)(\tilde{\nu})\right| .
$$

From (8.6), all properties listed in Lemma 8.2 persist if we change $\tilde{E}^{s}(\omega)$ and $\tilde{E}^{u}(\omega)$ to $E^{s}(\omega)$ and $E^{u}(\omega)$.

From (1) of Lemma 8.2, if we take $K(\omega, x)=c_{1}$ and $\beta(\omega, x)=-\log a$, then $\beta>0$ and $(\theta, \phi)$-invariant, $K(\omega, x)$ is tempered, and (8.1) and (8.2) hold.

From the uniform closeness of $\phi(t, \omega)$ and $\psi(t)$, the normal hyperbolicity property of $\mathcal{M}$ under $\psi(t)$ and the uniform closeness of $\tilde{\mathcal{M}}(\omega)$ and $\mathcal{M}$, we have for some $(\theta, \phi)$-invariant random variable $\alpha: \tilde{M} \rightarrow(0, \infty)$ and tempered random variable $K(\omega, x): \tilde{\mathcal{M}} \rightarrow[1, \infty)$ such that (8.3) holds. 
What we need to prove is that $r \alpha<\beta$. From (2) of Lemma 8.2 , $\alpha$ and $\beta$ can be chosen such that $r \alpha<\beta$.

Lemma 8.5. $E^{i}(\omega, m)$ is measurable.

Proof. In Sections 6 and 7, we proved the measurability of $E^{c}$. Here we only prove the measurability of $E^{u}$. For $E^{s}$, just follow exactly the same argument for $E^{u}$.

Take the orthogonal complement of $T_{(\omega, m)} \tilde{\mathcal{M}}$ in $T_{(\omega, m)} \tilde{\mathcal{W}}^{u}$ to get a normal bundle $\hat{E}^{u}(\omega, m)$. Since $T \tilde{\mathcal{M}}$ and $T \tilde{\mathcal{W}}^{u}$ are both measurable, $\hat{E}^{u}(\omega, m)$ is also measurable. Moreover, the angle between $\hat{E}^{u}(\omega, m)$ and $T_{(\omega, m)} \tilde{\mathcal{M}}$ is uniformly bounded away from 0. So by Lemma 8.4, all properties listed in Lemma 8.2 hold, even though $\hat{E}(\omega, m)$ may not close to $E^{u}$. So we can replace the bundle $\tilde{E}^{u}(\omega, m)$ in Lemma 8.3 by $\hat{E}^{u}(\omega, m)$ and still get the unique invariant bundle $E^{u}(\omega, m)$ by the same argument. In this way, the contraction mapping in the functional equation of $h(\omega, x)$ is measurable in $\omega$ and $C^{r-1}$ in $x$. As we obtained the measurability of $v$ in Proposition 5.4, we obtain the measurability of $h(\omega, x)$-the representation of $E^{u}(\omega, m)$. This completes the proof of the lemma.

By using Lemmas 8.3, 8.4 and 8.5 , we have Proposition 8.1

Summarizing Propositions 4.1, 5.3, 6.1, 6.3, 7.1, 7.2 and 8.1, gives Theorem 2.1,

\section{Persistence For OVERFlowing AND INFLOWING INVARIANT MANIFOldS}

In this section, we give the persistence of overflowing and inflowing manifolds. The proof of these results follows in the same fashion as the persistence of normally hyperbolic invariant manifolds with slight modifications.

Theorem 9.1. Assume that $\psi(t)(x)$ is a $C^{r}$ flow, $r \geq 1$, and has a compact, connected $C^{r}$ normally hyperbolic overflowing invariant manifold $\overline{\mathcal{M}}=\mathcal{M} \cup \partial \mathcal{M} \subset$ $\mathbb{R}^{n}$. Then there exists $\rho>0$ such that for any $C^{r}$ random flow $\phi(t, \omega, x)$ in $\mathbb{R}^{n}$, if

$$
\|\phi(t, \omega)-\psi(t)\|_{C^{1}}<\rho, \quad \text { for } t \in[0,1], \omega \in \Omega,
$$

then if $\alpha<r \beta, \phi(t, \omega)$ has a $C^{r}$ normally hyperbolic random overflowing invariant manifold $\tilde{\mathcal{M}}(\omega)$ such that for each $\omega \in \Omega, \tilde{\mathcal{M}}(\omega)$ is $C^{r}$ diffeomorphic to $\mathcal{M}$.

To prove this theorem, we enlarge the overflowing invariant manifold $\overline{\mathcal{M}}$ to $\overline{\mathcal{M}}_{1}:=\psi(1, \overline{\mathcal{M}})$ and $\overline{\mathcal{M}}_{2}:=\psi(2, \overline{\mathcal{M}})$ such that

$$
\overline{\mathcal{M}} \subset \mathcal{M}_{1} \subset \overline{\mathcal{M}}_{1} \subset \mathcal{M}_{2} \subset \overline{\mathcal{M}}_{2}
$$

where $M_{i}, i=1,2$, are the interiors of $\bar{M}_{i}$.

Following the same argument as in the proof of Theorem 2.1, one can construct $\tilde{\mathcal{M}}(\omega)$ near $\mathcal{M}_{1}$ in a tubular neighborhood $V$ of $\overline{\mathcal{M}}_{2}$ as a section of a normal bundle. Since the normal direction contains only the stable direction, there is no need to combine the center and the unstable direction. So the proof for the persistence actually is shorter.

We have the following theorem for the inflowing manifolds.

Theorem 9.2. Assume that $\psi(t)(x)$ is a $C^{r}$ flow, $r \geq 1$, and has a compact, connected $C^{r}$ normally hyperbolic inflowing invariant manifold $\overline{\mathcal{M}}=\mathcal{M} \cup \partial \mathcal{M} \subset$ $\mathbb{R}^{n}$. Then there exists $\rho>0$ such that for any $C^{r}$ random flow $\phi(t, \omega, x)$ in $\mathbb{R}^{n}$, if

$$
\|\phi(t, \omega)-\psi(t)\|_{C^{1}}<\rho, \quad \text { for } t \in[0,1], \omega \in \Omega,
$$


then if $\alpha<r \beta, \phi(t, \omega)$ has a $C^{r}$ normally hyperbolic random inflowing invariant manifold $\tilde{\mathcal{M}}(\omega)$ such that for each $\omega \in \Omega, \tilde{\mathcal{M}}(\omega)$ is $C^{r}$ diffeomorphic to $\mathcal{M}$.

Remark 1. For the overflowing case, if the normal direction contains both stable and unstable directions with 'similar' properties as those listed in Definition 2.4, then we also call it normally hyperbolic and an unstable manifold exists (see [F1] theorem 4) and persists under random perturbation.

Remark 2. For the inflowing case, if the normal direction contains both stable and unstable directions with 'similar' properties as those listed in Definition 2.4, then we also call it normally hyperbolic and a stable manifold exists and persists under random perturbation.

Remark 3. Generally, for the overflowing (inflowing) case, we have neither the existence of a stable (unstable) manifold of $\mathcal{M}$ nor the persistence of $\mathcal{M}$. So we do not have the persistence of the normally hyperbolicity, either. However, we have the following theorem about the persistence of normally hyperbolicity:

Theorem 9.3. Assume that $\psi(t)$ is a $C^{r}$ flow, $r \geq 1$, and has a compact, connected $C^{r}$ normally hyperbolic overflowing (inflowing) invariant manifold $\overline{\mathcal{M}}=\mathcal{M} \cup \partial \mathcal{M} \subset$ $\mathbb{R}^{n}$ with $\alpha<r \beta$, where $\mathcal{M}$ has both the stable and unstable manifolds $\mathcal{W}^{s}$ and $\mathcal{W}^{u}$. Then there exists $\rho>0$ such that for any random $C^{r}$ flow $\phi(t, \omega)$ in $\mathbb{R}^{n}$, if

$$
\|\phi(t, \omega)-\psi(t)\|_{C^{1}}<\rho, \quad \text { for } t \in[0,1], \omega \in \Omega,
$$

as long as $\phi(t, \omega)$ has a compact, connected $C^{r}$ random overflowing (inflowing) invariant manifold $\overline{\mathcal{M}}(\omega)$ with stable and unstable manifold $\tilde{\mathcal{W}}^{s}(\omega)$ and $\tilde{\mathcal{W}}^{u}(\omega)$ such that $\overline{\tilde{\mathcal{M}}}(\omega), \tilde{\mathcal{W}}^{s}(\omega)$ and $\tilde{\mathcal{W}}^{u}(\omega)$ are $C^{1}$ close to $\overline{\mathcal{M}}, \mathcal{W}^{s}$ and $\mathcal{W}^{u}$, respectively, then $\overline{\tilde{M}}(\omega)$ is normally hyperbolic with constant $\alpha<r \beta$.

The proof of this theorem follows the line of Section 8 .

\section{REFERENCES}

[A] L. Arnold, Random Dynamical Systems, Springer, New York (1998). MR1723992 (2000m:37087)

[BFGJ] P. Bates, P. Fife, R. Gardner, and C.K.R.T. Jones, Phase field models for hypercooled solidification, Physica D 104 (1997), 1-31. MR1447948 (97m:80011)

[BLZ1] P. Bates, K. Lu, and C. Zeng, Existence and persistence of invariant manifolds for semiflows in Banach space, Memoirs of the AMS, 135 (1998). MR.1445489 (99b:58210)

[BLZ2] P. Bates, K. Lu, and C. Zeng, Persistence of overflowing manifold for semiflow, Comm. Pure Appl. Math. 52 (1999), 983-1046. MR1686965 (2000f:37116)

[BLZ3] P. Bates, K. Lu, and C. Zeng, Invariant foliations for semiflows near a normally hyperbolic invariant manifold, Trans. Amer. Math. Soc., 352 (2000), 4641-4676. MR 1675237 (2001b:37031)

[BF] A. Bensoussan and F. Flandoli, Stochastic inertial manifold, Stochastics Stochastics Rep., 53 (1995), no. 1-2, 13-39. MR1380488 (97d:60102)

[Bx] P. Boxler, A stochastic version of center manifold theory, Probab. Theory Related Fields 83 (1989), no. 4, 509-545. MR1022628 (91d:58115)

[BK] M. Brin and Y. Kifer, Dynamics of Markov chains and stable manifolds for random diffeomorphisms, Ergodic Theory Dynam. Systems 7 (1987), no. 3, 351-374. MR.912374 (88k:58162)

[B] P. Brunovsky, Tracking invariant manifolds without differential forms, Acta Math. Univ. Comenianae Vol. 65, (1996), no. 1, 23-32. MR1422292 (97m:34100)

[CDLS] T. Caraballo, J. Duan, K. Lu, and B. Schmalfuss, Invariant manifolds for random and stochastic partial differential equations, Adv. Nonlinear Stud. 10 (2010), no. 1, 23-52. MR2574373 (2011f:60118) 
[CLR] T. Caraballo, J. Langa and J. C. Robinson, A stochastic pitchfork bifurcation in a reaction-diffusion equation, Proc. R. Soc. Lond. A 457 (2001), 2441-2453 MR.1857922 (2003a:60106)

[C] A. Carverhill, A formula for the Lyapunov numbers of a stochastic flow. Application to a perturbation theorem, Stochastics 14 (1985), no. 3, 209-226. MR800244 (86k:93141)

[CV] C. Castaing and M. Valadier. Convex Analysis and Measurable Multifunctions. LNM 580. Springer-Verlag, Berlin-Heidelberg-New York, 1977. MR0467310 (57:7169)

[DD] G. Da Prato and A. Debussche. Construction of stochastic inertial manifolds using backward integration, Stochastics Stochastics Rep. 59 (1996): no. 3-4, 305-324. MR.1427743 (98d:60121)

[D] S. Dahlke, Invariant manifolds for products of random diffeomorphisms, J. Dynam. Differential Equations 9 (1997), no. 2, 157-210. MR 1451289 (98m:58078)

[PJ] Pavel Drabek and Jaroslav Milota, Methods of Nonlinear Analysis. Applications to Differential Equations, Birkhäuser Verlag, Basel, 2007. MR2323436 (2008i:47134)

[DSL1] J. Duan, K. Lu, and B. Schmalfuss. Invariant manifolds for stochastic partial differential equations, Annals of Probability 31(2003), 2109-2135. MR2016614 (2004m:60136)

[DSL2] J. Duan, K. Lu and B. Schmalfuss, Smooth stable and unstable manifolds for stochastic evolutionary equations, J. Dynamics and Diff. Eqns, 16 (2004), 949-972. MR 2110052 (2005j:60124)

[F1] N. Fenichel, Persistence and smoothness of invariant manifolds for flows, Indiana Univ. Math. Journal 21 (1971), 193-226. MR0287106 (44:4313)

[F2] N. Fenichel, Asymptotic stability with rate conditions, Indiana Univ. Math. Journal 23 (1974), 1109-1137. MR0339276 (49:4036)

[F3] N. Fenichel, Asymptotic stability with rate conditions II, Indiana Univ. Math. Journal 26 (1977), 81-93. MR0426056 (54:14002)

[GLS] M. Garrido-Atienza, K. Lu, and B. Schmalfuss, Unstable invariant manifolds for stochastic PDEs driven by a fractional Brownian motion, J. Differential Equations 248 (2010), no. 7, 1637-1667. MR2593602 (2011a:60226)

[GC] T. V. Girya and I. D. Chueshov, Inertial manifolds and stationary measures for stochastically perturbed dissipative dynamical systems, Sb. Math. 186 (1995), no. 1, 29-45. MR 1641664 (99k:34134)

[H] J. Hadamard, Sur l'iteration et les solutions asymptotiques des equations differentielles, Bull. Soc. Math. France 29 (1901), 224-228.

[HPS] M. W. Hirsch, C.C. Pugh, and M. Shub, Invariant manifolds, Lecture Notes in Mathematics, 583, Springer-Verlag, New York, 1977. MR $0501173(58: 18595)$

[JK] C. K. R. T. Jones and N. Kopell, Tracking invariant manifolds with differential forms in singularly perturbed systems, J. Differential Equations 108 (1994), 64-88. MR1268351 (95c:34085)

[J] C. K. R. T. Jones, Stability of the travelling wave solution of the FitzHugh-Nagumo system, Trans. Amer. Math. Soc. 286 (1984), 431-469. MR760971 (86b:35011)

[K] T. Kato, Perturbation theory for linear operators, Die Grundlehren der mathematischen Wissenschaften, Band 132 Springer-Verlag New York, Inc., New York, 1966. MR0203473 $(34: 3324)$

[KSS] M. Krupa, B. Sandstede, and P. Szmolyan, Fast and Slow Waves in the FitzHughNagumo Equation, J. Differential Equations 133 (1997) 49-97. MR.1426757 (98e:35165)

[LiL] W. Li and K. Lu, Sternberg theorems for random dynamical systems, Comm. Pure Appl. Math. 58 (2005), no. 7, 941-988. MR2142880 (2006b:37099)

[LL] Z. Lian and K. Lu, Lyapunov Exponents and Invariant Manifolds for Infinite Dimensional Random Dynamical Systems in a Banach Space, Memoirs of the AMS. 206 (2010), no. 967, 106 pp. MR2674952 (2011g:37145)

[LQ] P-D. Liu and M. Qian, Smooth ergodic theory of random dynamical systems, Lecture Notes in Mathematics, 1606. Springer-Verlag, Berlin, 1995. MR 1369243 (96m:58139)

[LS] K. Lu and B. Schmalfuss, Invariant manifolds for stochastic wave equations, J. Differential Equations 236 (2007), no. 2, 460-492. MR2322020 (2009c:37063)

[MS] S.-E. A. Mohammed and M. K. R. Scheutzow, The stable manifold theorem for stochastic differential equations, The Annals of Probability 27 (1999), no. 2, 615-652. MR 1698943 (2001e:60121) 
[MZZ] S.-E. A. Mohammed, T. Zhang, and H. Zhao, The stable manifold theorem for semilinear stochastic evolution equations and stochastic partial differential equations, Memoirs of the American Mathematical Society 196 (2008), no. 917, 105 pp. MR2459571 (2010b:60175)

[R] D. Ruelle, Characteristic exponents and invariant manifolds in Hilbert spaces, Ann. of Math. 115 (1982), 243-290. MR647807 (83j:58097)

[S1] S. Schecter, Existence of Dafermos profiles for singular shocks, J. Differential Eqs. 205 (2004), 185-210. MR.2094383 (2005k:35269)

[S2] S. Schecter, Exchange lemmas. II. General exchange lemma, J. Differential Equations 245 (2008), no. 2, 411-441. MR2428005 (2009h:37052)

[S] B. Schmalfuss, A random fixed point theorem and the random graph transformation, Journal of Mathematical Analysis and Applications, 225 (1998), no. 1, 91-113. MR.1639297 (99i:47118)

[WD] W. Wang and J. Duan, A dynamical approximation for stochastic partial differential equations, J. Math. Phys. 48 (2007), no. 10, 102701, 14pp. MR2362785 (2009d:60211)

[W] T. Wanner, Linearization random dynamical systems. In C. Jones, U. Kirchgraber and H. O. Walther, editors, Dynamics Reported, Vol. 4, 203-269, Springer-Verlag, New York, 1995. MR 1346499 (96m:34084)

[W] H. Whitney, Differential manifolds, Ann. of Math. 37 (1936), 645-680. MR1503303

Department of Mathematics, Brigham Young University, Provo, Utah 84602

E-mail address: liji@math.byu.edu

Current address: Institute for Mathematics and its Application, University of Minnesota, Minneapolis, Minnesota 55455

E-mail address: liji@ima.umn.edu

Department of Mathematics, Brigham Young University, Provo, Utah 84602 - And School of Mathematics, Sichuan University, Chengdu, People's Republic of China

E-mail address: klu@math.byu.edu

Department of Mathematics, Michigan State University, East Lansing, Michigan 48824

E-mail address: bates@math.msu.edu 